\title{
Low-Threshold Mechanosensitive VGLUT3-Lineage Sensory Neurons Mediate Spinal Inhibition of Itch by Touch
}

\author{
${ }^{\circledR}$ Kent Sakai, ${ }^{1}{ }^{\circledR}$ Kristen M. Sanders, ${ }^{1}{ }^{\circledR}$ Shing-Hong Lin, ${ }^{2,3}$ Darya Pavlenko, ${ }^{1}$ Hideki Funahashi, ${ }^{1,4}$ Taisa Lozada, ${ }^{1}$ \\ (D) Shuanglin Hao, ${ }^{5}$ Chih-Cheng Chen, ${ }^{2}$ and ${ }^{\text {DD Tasuku Akiyama }}{ }^{1}$ \\ ${ }^{1}$ Dr. Phillip Frost Department of Dermatology \& Cutaneous Surgery and Miami Itch Center, University of Miami Miller School of \\ Medicine, Miami, Florida 33136, ${ }^{2}$ Institute of Biomedical Sciences, Academia Sinica, Taipei, 115, Taiwan, ${ }^{3}$ Department of Neurobiology, \\ Dana-Farber Cancer Institute, Harvard Medical School, Boston, Massachusetts 02115, ${ }^{4}$ Department of Psychiatry, Faculty of Medicine, \\ University of Miyazaki, Miyazaki City, Miyazaki 5200, Japan, and ${ }^{5}$ Department of Anesthesiology, University of Miami Miller School of \\ Medicine, Miami, Florida, 33136
}

Innocuous mechanical stimuli, such as rubbing or stroking the skin, relieve itch through the activation of low-threshold mechanoreceptors. However, the mechanisms behind this inhibition remain unknown. We presently investigated whether stroking the skin reduces the responses of superficial dorsal horn neurons to pruritogens in male C57BL/6J mice. Single-unit recordings revealed that neuronal responses to chloroquine were enhanced during skin stroking, and this was followed by suppression of firing below baseline levels after the termination of stroking. Most of these neurons additionally responded to capsaicin. Stroking did not suppress neuronal responses to capsaicin, indicating state-dependent inhibition. Vesicular glutamate transporter 3 (VGLUT3)-lineage sensory nerves compose a subset of low-threshold mechanoreceptors. Stroking-related inhibition of neuronal responses to chloroquine was diminished by optogenetic inhibition of VGLUT3-lineage sensory nerves in male and female Vglut3-cre/NpHR-EYFP mice. Conversely, in male and female Vglut3-cre/ChR2-EYFP mice, optogenetic stimulation of VGLUT3-lineage sensory nerves inhibited firing responses of spinal neurons to pruritogens after the termination of stimulation. This inhibition was nearly abolished by spinal delivery of the $\boldsymbol{\kappa}$-opioid receptor antagonist nor-binaltorphimine dihydrochloride, but not the neuropeptide Y receptor Y1 antagonist BMS193885. Optogenetic stimulation of VGLUT3-lineage sensory nerves inhibited pruritogen-evoked scratching without affecting mechanical and thermal pain behaviors. Therefore, VGLUT3-lineage sensory nerves appear to mediate inhibition of itch by tactile stimuli.

Key words: itch; mice; optogenetics; spinal cord; touch; VGLUT3

Significance Statement

Rubbing or stroking the skin is known to relieve itch. We investigated the mechanisms behind touch-evoked inhibition of itch in mice. Stroking the skin reduced the activity of itch-responsive spinal neurons. Optogenetic inhibition of VGLUT3-lineage sensory nerves diminished stroking-evoked inhibition, and optogenetic stimulation of VGLUT3-lineage nerves inhibited pruritogen-evoked firing. Together, our results provide a mechanistic understanding of touch-evoked inhibition of itch.

\section{Introduction}

Itch is often characterized by its relationship to scratching and other noxious counterstimuli, which are well known to inhibit itch. Less attention has been paid to the relationship between

\footnotetext{
Received Jan. 13, 2020; revised July 7, 2020; accepted July 20, 2020.

Author contributions: K.S., K.M.S., D.P., H.F., and T.L. performed research; K.S. and T.A. wrote the first draft of the paper; K.S., K.M.S., S.-H.L., S.H., C.-C.C., and T.A. edited the paper; K.S., K.M.S., and T.A. wrote the paper; K.S., S.-H.L., S.H., C.-C.C., and T.A. analyzed data; S.-H.L., C.-C.C., and T.A. designed research.

The authors declare no competing financial interests.

This work was supported by National Institutes of Health Grants R00AR063228 and R01AR074062 to T.A. and Grant R01DA047157 to S.H., and the Ministry of Science and Technology, Taiwan MOST 108-2320-B-001021-MY3 to C.-C.C. We thank Kevin Johnson (University of Miami) for generous technical support.

Correspondence should be addressed to Tasuku Akiyama at takiyama@miami.edu.

https://doi.org/10.1523/JNEUROSCI.0091-20.2020

Copyright $\odot 2020$ the authors
}

itch and innocuous stimuli, such as touch. Rubbing or stroking of the skin is commonly used to relieve itch in sensitive areas (e.g., the eyes or nose) and is also frequently used by chronic itch patients (Stander et al., 2007). For example, onethird of hemodialysis patients suffering from chronic itch reported that rubbing their skin combated itch (Hayani et al., 2016), and many patients with urticaria rub rather than scratch (Sabroe and Greaves, 2004). Rubbing or stroking the skin activates low-threshold mechanoreceptors (LTMRs), which are classified as A $\beta$-LTMR, A $\delta$-LTMR, and C-LTMR based on their conduction velocity (Abraira and Ginty, 2013). These fibers input to laminae II-IV of the spinal dorsal horn (Abraira et al., 2017). While it has been proposed that rubbing the skin activates itch inhibitory neuronal circuits within the spinal cord (Yosipovitch et al., 2003; Sabroe and Greaves, 
2004), the mechanisms behind itch relief by innocuous mechanical stimuli are virtually unknown.

Many peripheral sensory neurons express vesicular glutamate transporters (VGLUTs) 1-3, which are vesicular membranebound proteins that transport the excitatory neurotransmitter glutamate into presynaptic vesicles (Fremeau et al., 2004). VGLUT3-lineage sensory nerves consist of at least three subgroups, including (1) A-fibers innervating Merkel cells (a subset of A $\beta$-LTMR fibers), (2) C-fibers expressing tyrosine hydroxylase (TH) (TH; C-LTMR fibers), and (3) C-fibers not expressing TH (Seal et al., 2009; Lou et al., 2013; Draxler et al., 2014; Griffith et al., 2019), and are involved in sensory modulation in the spinal cord. Multiple lines of evidence suggest that VGLUT3-lineage neurons may mediate touch inhibition of itch. First, A-fibers innervating Merkel cells have been shown to inhibit touchevoked itch, presumably through activation of $\mathrm{NPY}^{+}$inhibitory interneurons (Feng et al., 2018; Sakai and Akiyama, 2019). Second, C-LTMR fibers inhibit signaling from slowly conducting C-fibers (e.g., heat-sensitive C-fibers) in the rat spinal cord (Lu and Perl, 2003). Despite this evidence, the role of VGLUT3-lineage sensory nerves in the processing of itch in the spinal cord remains poorly understood.

We hypothesized that innocuous mechanical stimuli would inhibit itch signaling in the spinal cord. First, we performed in vivo single-unit electrophysiological recording from spinal neurons to investigate whether the increased firing rates of pruritogen-responsive neurons are reduced by stroking the skin. We further hypothesized that VGLUT3-lineage primary afferents would mediate the relief of itch by innocuous mechanical stimuli. To directly activate or silence peripheral VGLUT3-lineage sensory nerves, we generated mice that express the excitatory opsin channelrhodopsin-2 or the inhibitory opsin halorhodopsin in VGLUT3-lineage sensory nerves. Using these mice, we tested whether innocuous mechanical stimuli-evoked inhibition could be attenuated or reversed by optogenetic inhibition of VGLUT3lineage sensory nerves. Finally, we addressed the role of VGLUT3lineage sensory nerves in spinal itch modulation by determining whether optogenetic stimulation of VGLUT3-lineage sensory nerves reduced the firing rates of pruritogen-responsive neurons during pruritogen stimulation.

\section{Materials and Methods}

Mice. All procedures were approved by the Institutional Animal Care and Use Committee of the University of Miami. All mice were group-housed (2-5 per cage), given standard food and water ad libitum, and maintained under a $12 \mathrm{~h}$ light/dark cycle (6:00 lights on, 18:00 lights off). All mice were at least 8 weeks old at the time of experiments. Adult male and female mice were randomly assigned to experimental conditions. Mice were typically used for a battery of behavioral tests, with a 1 week break between each test. Before behavior testing, mice were habituated twice to each behavioral test apparatus for the equivalent of the recording time.

Vglut3-Cre mice ( $\mathrm{Tg}($ Slc17a8-icre)1Edw, The Jackson Laboratory) (Grimes et al., 2011) were crossed with the following strains: Ai32 mice expressing Cre-dependent channelrhodopsin-2 (ChR2)-EYFP (B6;129SGt(ROSA)26Sor ${ }^{\text {tm } 32\left(C A G-C O P 4^{*} H 134 R / E Y F P\right) H z e} / J$, The Jackson Laboratory (Madisen et al., 2012); Ai39 mice expressing Cre-dependent halorhodopsin (NpHR)-EYFP (B6;129S-Gt(ROSA)26Sor tm39(CAG-hop/EYFP)Hze $/ J$, The Jackson Laboratory) (Madisen et al., 2012); and Ai14 mice expressing robust Cre-dependent tdTomato fluorescence (B6;129S6-Gt(ROSA)

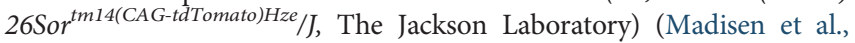
2010). Vglut3-cre/ChR2-EYFP and Vglut3-cre/NpHR-EYFP mice were used for electrophysiology recording and immunohistochemistry. Vglut3-cre;TdTomato mice were used for immunohistochemistry.
Table 1. Stroking and scratching devices

\begin{tabular}{llll}
\hline & Strength $(\mathrm{mN})$ & Width $(\mathrm{mm})$ & Speed $(\mathrm{cm} / \mathrm{s})$ \\
\hline Cotton swab & 0.21 & 4 & 2 \\
Thin brush & $49-64$ & 2.5 & 2 \\
Thick brush & $24-35$ & 6 & 2 \\
\hline
\end{tabular}

C57BL/6J mice (male, 8-16 weeks, 22-38 g) were obtained from The Jackson Laboratory to perform electrophysiology recordings.

In vivo single-unit recording from lumbar spinal cord. Single-unit recording from the lumbar spinal cord was conducted as previously detailed (Cuellar et al., 2004; Akiyama et al., 2009). Briefly, mice were anesthetized with sodium pentobarbital $(80 \mathrm{mg} / \mathrm{kg}$, i.p.). The overlying muscles were dissected, and the L2-L4 lumbar spinal cord was exposed by laminectomy. A tungsten microelectrode (FHC) was driven into the superficial spinal cord by hydraulic microdrive (David Kopf) to record extracellular single-unit activity, which was amplified and digitized using Powerlab (AD Instruments) and Spike2 (CED) software. The spinal cord was continually superfused with ACSF consisting of the following (in m): $117 \mathrm{NaCl}, 3.6 \mathrm{KCl}, 2.5 \mathrm{CaCl}_{2}, 1.2 \mathrm{MgCl}_{2}, 1.2 \mathrm{NaH}_{2} \mathrm{PO}_{4}, 25$ $\mathrm{NaHCO}_{3}$, and 11 glucose, equilibrated with $95 \% \mathrm{O}_{2} / 5 \% \mathrm{CO}_{2}$ at $37^{\circ} \mathrm{C}$.

A chemical search strategy (Jinks and Carstens, 2000) was used to isolate units in the superficial dorsal horn. In this study, chloroquine diphosphate salt (100 $\mu \mathrm{g} / 1 \mu \mathrm{l}$ in PBS; Sigma Millipore) was used as the search stimulus because chloroquine-responsive spinal neurons largely overlap with bombesin-responsive neurons postulated to signal itch (Sun et al., 2009; Mishra and Hoon, 2013; Akiyama et al., 2014a). Using a $30.5 \mathrm{G}$ needle connected to a Hamilton microsyringe, a small volume $(\sim 0.1 \mu \mathrm{l})$ was microinjected (i.d.) into the dorsal hindpaw skin, and the recording electrode was positioned to isolate an action potential in the superficial dorsal horn ( $300 \mu \mathrm{m}$ from the surface) that had ongoing activity. After the ongoing activity subsided, $\sim 1 \mu \mathrm{l}$ of chloroquine was injected through the same needle. Only units exhibiting a $>3 \mathrm{SD}$ increase in firing rate were selected for further study. In each experiment, some or all of the following additional stimuli were delivered to the dorsal hindpaw: innocuous stroking, pinch, i.d. PBS, serotonin $(10 \mu \mathrm{g} / 1 \mu \mathrm{l}$ in PBS; Alfa Aesar), histamine $(50 \mu \mathrm{g} / 1 \mu \mathrm{l}$ in PBS; Sigma Millipore), 7\% Tween 80 in PBS (vehicle for capsaicin), and capsaicin $(30 \mu \mathrm{g} / 1 \mu \mathrm{l}$ in PBS-Tween; Sigma Millipore).

During the time that the unit exhibited a relatively stable level of firing following i.d. injection of pruritogen or capsaicin (usually 3-5 min after injection), stroking stimuli were successively delivered to the dorsal hindpaw. Stroking was accomplished by moving a cotton swab or different sized brushes (Table 1) in a back-and-forth motion across the dorsal hindpaw skin at a constant frequency of $2 \mathrm{~Hz}$, excursion of $6 \mathrm{~mm}$, and duration of 20-30 s.

In A-fiber silencing experiments, flagellin ( $10 \mathrm{pmol}$; Enzo Life Sciences) and QX-314 (120 nmol; Tocris R\&D Systems) (Xu et al., 2015) were injected i.d. into the shaved dorsal hindpaw. One hour after the i.d. injection, mice were used for electrophysiology experiments. Two to $3 \mathrm{~h}$ after the i.d. injection, stroking stimuli were delivered during chloroquine responses.

In Vglut3-cre/ChR2-EYFP mouse experiments, blue or green light stimulation $(20 \mathrm{~Hz}, 7 \mathrm{~mW})$ was delivered to the dorsal hindpaw instead of stroking stimuli. In Vglut3-cre/NpHR-EYFP mouse experiments, blue or green light stimulation (constant, $7 \mathrm{~mW}$ ) was delivered during stroking stimuli. In some experiments, either nor-binaltorphimine dihydrochloride (100 nм in ACSF; Santa Cruz Biotechnology) or BMS193885 (100 nM in ACSF; Tocris R\&D Systems) was delivered to the spinal cord for $30 \mathrm{~s}$, followed by switching back to ACSF alone.

At the conclusion of recording, an electrolytic lesion was made. The spinal cord was fixed in $4 \% \mathrm{PFA}$, and $50 \mu \mathrm{m}$ sections were cut and mounted on slides for microscopic verification of the lesion site.

In vivo single-unit recording from DRGs. Mice were anesthetized with urethane (1.5 $\mathrm{g} / \mathrm{kg}$, i.p.). A 3-cm-long skin incision was made aseptically at the midline of the lower back. The overlying muscles were dissected and removed from the L4 -L5 spinous processes. To expose the L4 or L5 DRGs, a small dorsolateral laminectomy was performed by removing the processus accessorius and part of the processus 
transversus. To loosen the neuronal somata from adjacent cells, collagenase P ( $3 \mathrm{mg} / \mathrm{ml}$; Sigma Millipore) was applied to the DRG, incubated for $5 \mathrm{~min}$, and washed 3 times with ACSF. Collagenase application was repeated 4 times. The DRG was continually superfused with ACSF. A tungsten microelectrode was driven into the DRG by hydraulic microdrive to record extracellular single-unit activity, which was amplified and digitized using Powerlab and Spike2 software.

Units were categorized as LTMRs if they responded maximally to light touch. To estimate conduction velocity, transcutaneous electrical stimuli $(0-8 \mathrm{~mA}, 2 \mathrm{~ms})$ were delivered from a pulse stimulator (model 2100; A-M Systems) to receptive fields. Units were further classified by conduction velocity as follows: A $\beta-(>9.0 \mathrm{~m} / \mathrm{s}), \mathrm{A} \delta-(1.0-9.0 \mathrm{~m} / \mathrm{s})$, and C-fibers $(<1.0 \mathrm{~m} / \mathrm{s})$ (McIlwrath et al., 2007; Wetzel et al., 2007; Woodbury and Koerber, 2007). Each unit was retested with brush and pinch stimuli to establish a baseline response level.

To verify A $\beta$-fiber silencing, either QX-314 (24 or $120 \mathrm{nmol}$ ) or a mix of flagellin (10 pmol) and QX-314 (24 or $120 \mathrm{nmol})$ was microinjected id $(10 \mu$ l volume) within the mechanosensitive receptive field. Units were then tested with brush and pinch stimuli at 30,60,120, and 240 min after injection. In some experiments, Vglut3-cre/ChR2-EYFP mice were used to validate the optogenetic stimulation of VGLUT3-lineage nerves in vivo. In these experiments, blue light was delivered to the skin surface.

Immunohistochemistry. Mice were killed under sodium pentobarbital anesthesia, and the skin was immediately dissected. Skin was fixed in Zamboni fixative solution (Newcomer Supply) followed by 30\% sucrose, frozen in optimal cutting temperature compound (Tissue-Tek, Sakura Finetek), and cut in $40 \mu \mathrm{m}$ sections on a cryostat.

Skin sections from Vglut3-cre/ChR2-EYFP and Vglut3-cre/NpHREYFP mice were blocked with $5 \%$ goat serum and $0.2 \%$ Triton X-100 in PBS for $2 \mathrm{~h}$ at room temperature, then immunostained with chicken anti-GFP antibody (1:1000; Aves Labs) at $4^{\circ} \mathrm{C}$ overnight, followed by incubation with the corresponding secondary antibody conjugated with AlexaFluor-488 (1:300) at $37^{\circ} \mathrm{C}$ for $30 \mathrm{~min}$. Then, the sections were immunostained with rabbit anti-VGLUT3 antibody (1:500) followed by the corresponding secondary antibody conjugated with AlexaFluor-594 (1:300; Invitrogen) at $37^{\circ} \mathrm{C}$ for $30 \mathrm{~min}$.

Skin sections from Vglut3-cre/TdTomato mice were blocked with 5\% donkey serum and $0.2 \%$ Triton X-100 in PBS, then immunostained with rabbit anti-PGP9.5 (1:200; EMD Millipore), rabbit anti-calcitonin gene-related peptide (CGRP) (1:300; Peninsula Laboratories), rabbit anti-P2X3 (1:200; Neuromics), or rabbit anti-Neurofilament H (NFH) antibody (1:200, Sigma Millipore) at $4^{\circ} \mathrm{C}$ overnight, followed by incubation with the corresponding secondary antibody conjugated with AlexaFluor- $488(1: 300)$ at $37^{\circ} \mathrm{C}$ for $30 \mathrm{~min}$.

For immunostaining of VGLUT3, skin was dissected from Vglut3cre/TdTomato mice and frozen in optimal cutting temperature compound (Tissue-Tek), and cut in $40 \mu \mathrm{m}$ sections on a cryostat. Skin sections were fixed in Zamboni fixative solution and blocked with 5\% donkey serum and $0.2 \%$ Triton X-100 in PBS for $2 \mathrm{~h}$ at room temperature, then immunostained with rabbit anti-VGLUT3 antibody (1:500, Synaptic Systems) followed by the corresponding secondary antibody conjugated with AlexaFluor-488 (1:300; Invitrogen) at $37^{\circ} \mathrm{C}$ for $30 \mathrm{~min}$. Then, the sections were immunostained with rabbit anti-red fluorescent protein antibody conjugated with biotin (1:500; Rockland Immunochemicals) at $4^{\circ} \mathrm{C}$ overnight, followed by incubation with the streptavidin conjugated with AlexaFluor-594 (1:100; Invitrogen) at $37^{\circ} \mathrm{C}$ for $30 \mathrm{~min}$.

All sections were mounted on slides with Vectashield Hardset Antifade Mounting Medium with DAPI (Vector Laboratories). Images were obtained with a CTR6000 fluorescence microscope with $20 \times$ objective magnification (Leica Microsystems).

Optogenetics. For optic stimulation or inhibition, a fiber-optic cable was connected to an LED light source (Prizmatix) that delivered blue light (460 nm wavelength) and green light $(520 \mathrm{~nm}$ wavelength). For Vglut3-cre/ChR2-EYFP mice, blue light $(20 \mathrm{~Hz})$ was applied to the skin to stimulate ChR2-expressing VGLUT3-lineage nerve endings during behavioral tests and electrophysiology experiments. Green light $(20 \mathrm{~Hz})$ was used as a control. For Vglut3-cre/NpHR-EYFP mice, a constant green light was applied to the skin to inhibit NpHR-expressing VGLUT3-lineage nerve endings during electrophysiology experiments. A constant blue light was used as a control.
Scratching behavior. Fur on the rostral back was shaved with electric clippers 1 week before the scratching test. After $30 \mathrm{~min}$ habituation to a Plexiglas recording arena $\left(15 \times 15 \times 15 \mathrm{~cm}^{3}\right)$, histamine $(50 \mu \mathrm{g} / 10 \mu \mathrm{l})$, serotonin hydrochloride $(10 \mu \mathrm{g} / 10 \mu \mathrm{l})$, or chloroquine diphosphate salt $(100 \mu \mathrm{g} / 10 \mu \mathrm{l})$ was injected i.d. into the shaved rostral back skin. The light was delivered from the center of the ceiling of the recording arena. Behavior was recorded for $30 \mathrm{~min}$ with green light, blue light, or no light stimulation of the shaved skin (power: $2.5-7 \mathrm{~mW}$, frequency: $20 \mathrm{~Hz}$, distance between light and skin surface: $1-5 \mathrm{~cm}$ ). The number of scratch bouts was analyzed in $5 \mathrm{~min}$ bins by a trained observer blinded to the treatment condition. One scratch bout was defined as one or more rapid back-and-forth hindpaw motions directed toward and contacting the injection site, ending with licking or biting of the toes or placement of the hindpaw on the floor. Hindpaw movements directed away from the injection site (e.g., ear-scratching) and grooming movements were not counted.

Hargreaves test. After $120 \mathrm{~min}$ habituation to the Hargreaves arena, the plantar surface of the hindpaws was exposed to 5 heat trials along with green light, blue light, or no light stimulation $(4 \mathrm{~mW}, 20 \mathrm{~Hz})$. Mice were assessed for paw withdrawal latencies. The beam active and idle intensities were 38 and 5, respectively. A cutoff of time of $10 \mathrm{~s}$ was used to prevent excessive tissue damage.

Hindpaw withdrawal test. After $120 \mathrm{~min}$ habituation to a perforated metal floor, the plantar surface of the hindpaws was tested with a series of von Frey filaments (0.07-4 g) along with green light, blue light, or no light stimulation $(4 \mathrm{~mW}, 20 \mathrm{~Hz})$. The strength $(\mathrm{g})$ of the von Frey filament, which induced paw withdrawal, was noted for each stimulus.

Statistical analysis. Results are presented as mean \pm SEM. For comparison between two groups, a two-tailed Student's $t$ test was used. For comparison among more than two groups, a one-way or twoway repeated-measures ANOVA followed by Tukey multiple comparisons test was used. Statistical significance was set at $p<0.05$ for all tests. All statistical analyses and graphs were made using GraphPad Prism8.

\section{Results}

\section{Stroking of the skin inhibits responses of spinal neurons to the itch stimulus chloroquine, but not the pain stimulus capsaicin}

To test whether stroking the skin inhibits spinal firing evoked by the itch stimulus chloroquine, we performed in vivo single-unit recording from $\mathrm{C} 57 \mathrm{BL} / 6 \mathrm{~J}$ mice. We used a chemical search strategy to isolate chloroquine-responsive units (Akiyama et al., 2009, 2014b). Next, chloroquine was microinjected into the dorsal hindpaw. During the chloroquine-evoked response, we stroked the dorsal hindpaw skin with a cotton swab, a thin brush, or a thick brush (Table 1).

An example unit is shown in Figure 1. This unit was located in the superficial dorsal horn and responded to i.d. injection of chloroquine (Fig. 1A). During the chloroquine-evoked response, stroking with the cotton swab, the thin brush, or the thick brush further excited the neuron (Fig. $1 A-D$ ). After stroking with the thick brush, the firing was decreased compared with prestroking. Neither PBS (vehicle for chloroquine) nor PBS Tween (vehicle for capsaicin) elicited a response (Fig. 1E). Following vehicle injections, the two brushes, but not the cotton swab, excited the unit. An i.d. injection of capsaicin excited the unit, and capsaicin-evoked firing was not inhibited by stroking with the cotton swab, the thin brush, or the thick brush (Fig. $1 F-I$ ).

We identified 10 chloroquine-responsive units in the dorsal horn. Unit recording sites were in the superficial dorsal horn based on micrometer depth (mean \pm SEM, 95.3 $\pm 25.4 \mu \mathrm{m}$ ). For most units, the location was confirmed by histologic identification of lesion sites (Fig. $2 A$, inset). PBS vehicle did not increase mean unit firing (Fig. 2A). As expected, mean firing immediately 


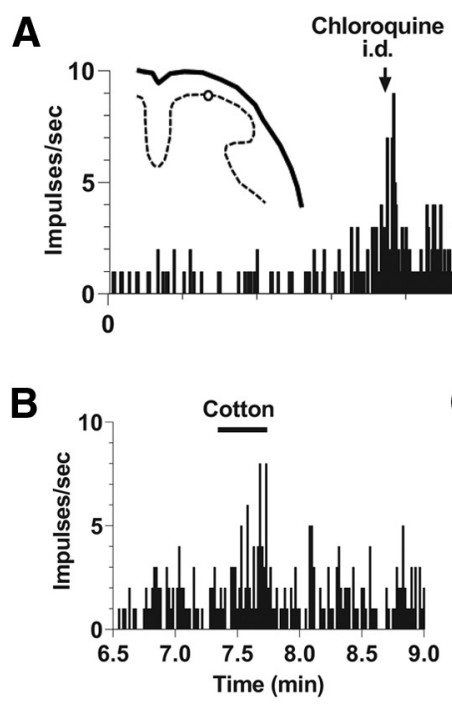

E
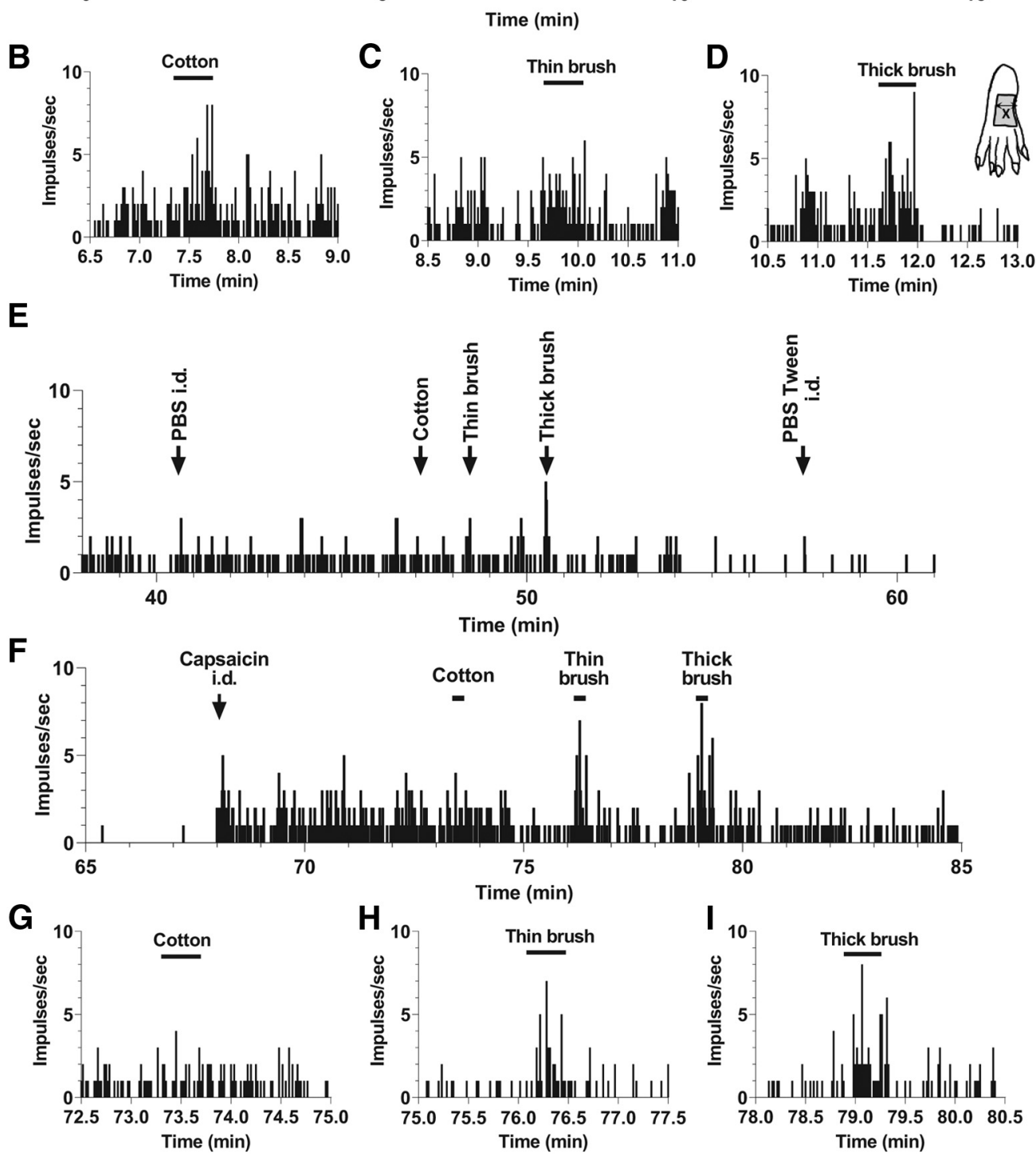

Time (min)
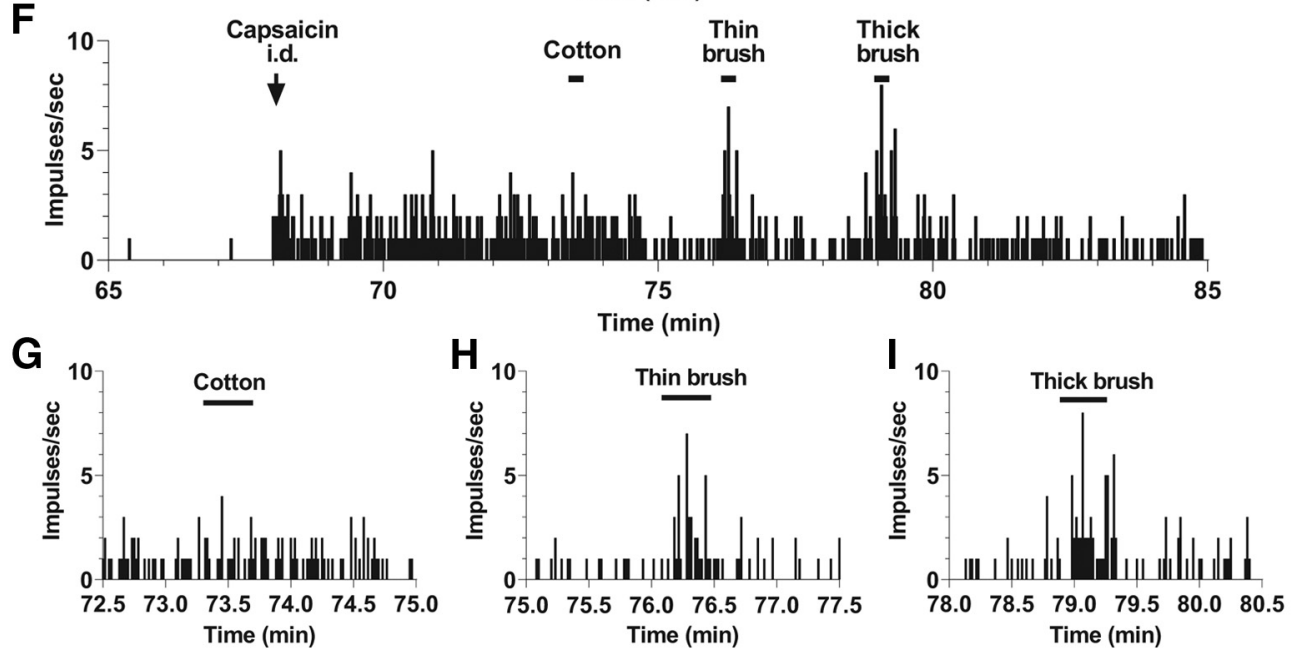

Figure 1. Example in vivo single-unit electrophysiological recording from a chloroquine-responsive unit in the spinal cord in $(57 \mathrm{BL} / 6 \mathrm{~J}$ mice. $\boldsymbol{A}$, This unit displayed a positive response over baseline to intradermal chloroquine. During chloroquine-evoked firing, the unit showed an enhanced response to $(\boldsymbol{B})$ stroking with a cotton swab, $(\boldsymbol{C})$ stroking with a thin brush, and $(\boldsymbol{D})$ stroking with a thick brush. The unit's firing rate was decreased after stroking with the thick brush. $\boldsymbol{E}$, The same unit did not display a positive response over baseline to intradermal PBS (vehicle for chloroquine), or PBS-Tween (vehicle for capsaicin). Following vehicle injections, the unit displayed a positive response to the thin brush and thick brush, but not to the cotton swab. $\boldsymbol{F}$, The unit displayed a positive response over baseline to intradermal capsaicin. During capsaicin-evoked firing, the unit had no response to $(\boldsymbol{G})$ the cotton swab but showed a positive response to $(\boldsymbol{H})$ the thin brush and $(I)$ the thick brush. $\boldsymbol{A}$, Inset, Recording site. $\boldsymbol{D}$, Inset, Injection/stroking site.

after chloroquine injection was significantly greater compared with the pre-injection level $\left(t_{(9)}=3.06, p=0.014\right.$; Fig. $\left.2 B\right)$.

During the chloroquine-evoked response, mean firing was enhanced during stroking with the cotton swab, thin brush, or thick brush (cotton swab: from $2.35 \pm 0.62$ to $4.17 \pm 0.9$ impulses/ $\mathrm{s}$; thin brush: from $2.69 \pm 0.69$ to $6.41 \pm 1.51 \mathrm{impulses} / \mathrm{s}$; thick brush: from $2.59 \pm 0.72$ to $6.83 \pm 1.65$ impulses/s; Fig. $2 E-H)$. The mean firing rate after thick brush $(1.03 \pm 0.31$ impulses/s) was significantly lower than the mean firing rate before thick brush $\left(2.59 \pm 0.72\right.$ impulses $/ \mathrm{s} ; F_{(1.21,10.85)}=11.24, p=0.005$; Tukey's multiple comparison test: $p=0.049$; Fig. $2 E, G, H)$. Neither the cotton swab nor the thin brush affected the mean firing rate after stroking (cotton: from $2.35 \pm 0.62$ to $2.13 \pm 1.72$ impulses/s; thin brush: from $2.69 \pm 0.69$ to $2.55 \pm 1.02$ impulses/s; Fig. $2 E-G$ ).

Of the 10 recorded units, 9 (90\%) also responded to capsaicin (Fig. 2C,D). Mean unit firing immediately after capsaicin injection was significantly greater compared with the pre-injection level $\left(t_{(8)}=4.77, p=0.0014\right.$; Fig. $\left.2 D\right)$, whereas PBS Tween vehicle 

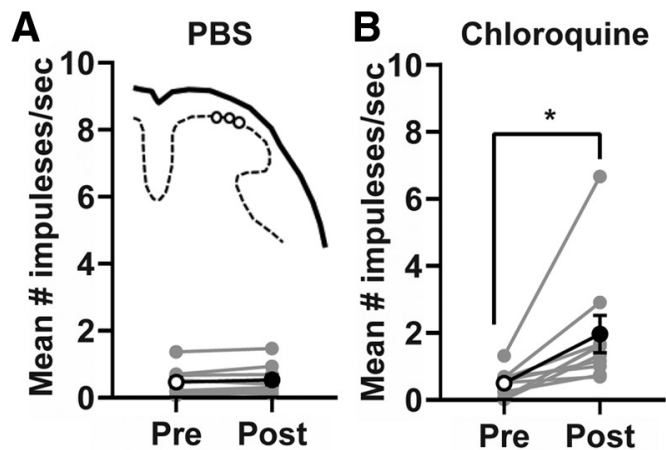

\section{E}
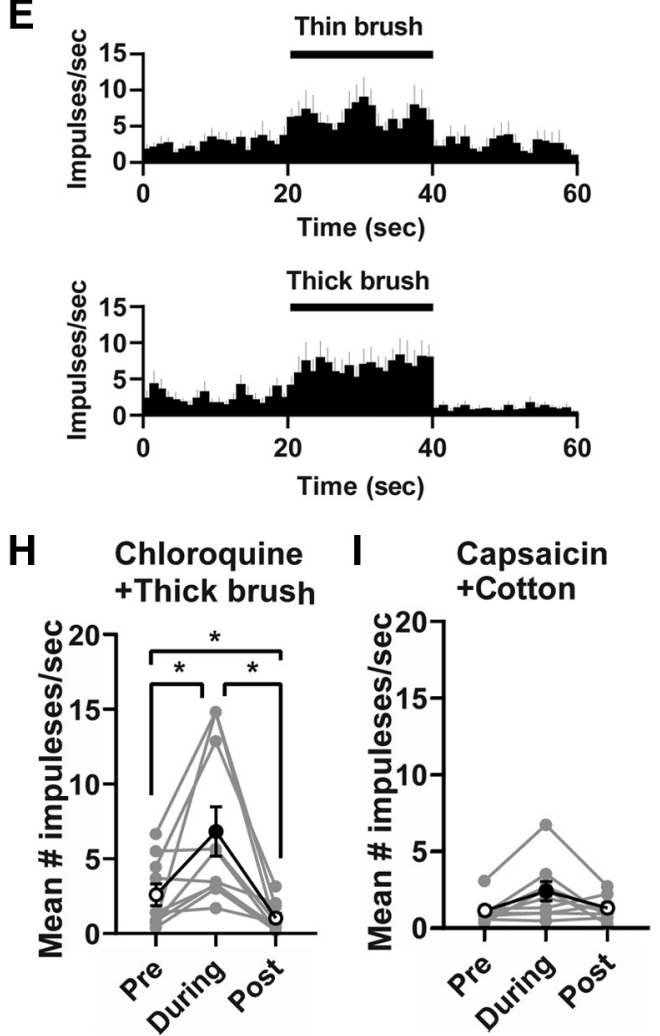
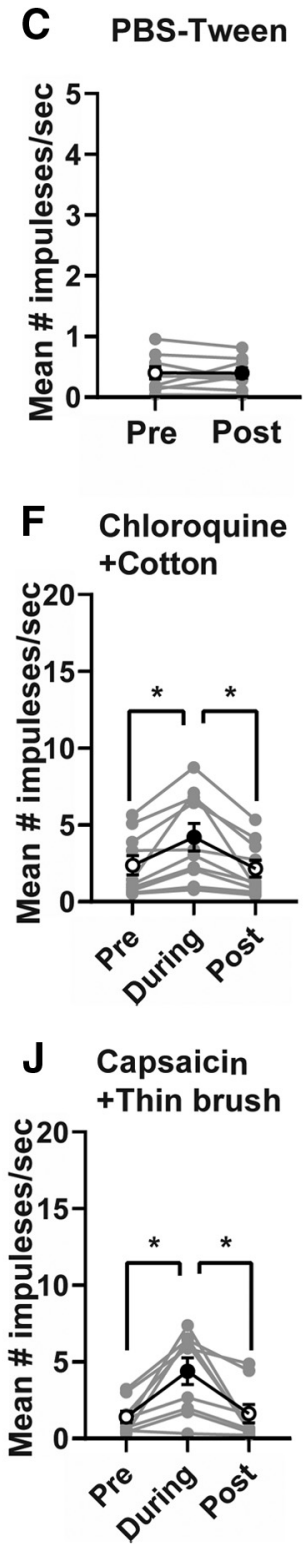
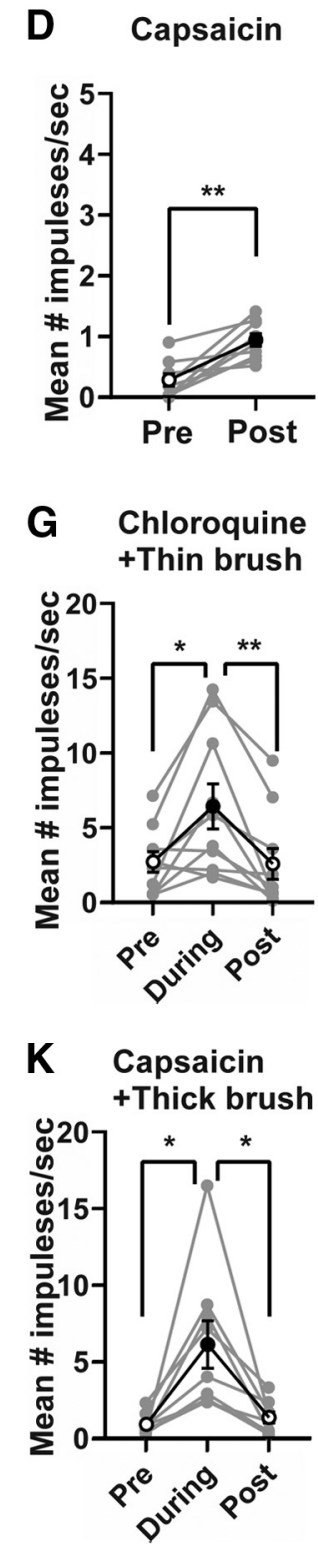

Figure 2. Stroking the skin with a thick brush reduced chloroquine-evoked firing in the spinal cord in C57BL/6J mice. $A$, Average firing of 10 units recorded from the superficial dorsal horn of spinal cord before (pre) and after (post) intradermal PBS. Inset, Recording sites. $\boldsymbol{B}$, Same as in $\boldsymbol{A}$, for chloroquine injection. $\boldsymbol{C}$, Same as in $\boldsymbol{A}$, for PBS-Tween injection (vehicle control for capsaicin injection). $\boldsymbol{D}$, Same as in $\boldsymbol{A}$, for capsaicin injection. $\boldsymbol{E}$, Averaged peristimulus time histogram (bins: $1 \mathrm{~s}$ ) of activity following i.d. chloroquine in 10 superficial dorsal horn units before, during, and after stroking with the thin or thick brushes. Horizontal lines indicate duration of stroking. $\boldsymbol{F}$, Individual (gray lines) and mean (black line) responses of superficial dorsal horn units following i.d. chloroquine, before (pre), during, and after (post) stroking with a cotton swab. $\boldsymbol{G}$, Same as in $\boldsymbol{F}$, for thin brush. $\boldsymbol{H}$, Same as in $\boldsymbol{F}$, for thick brush. $\boldsymbol{I}$, Individual (gray lines) and mean (black line) responses of superficial dorsal horn units following i.d. capsaicin before, during, and after stroking with the cotton swab. $\boldsymbol{J}$, Same as in $\boldsymbol{I}$, for thin brush. $\boldsymbol{K}$, Same as in $\boldsymbol{I}$, for thick brush. Data are mean \pm SEM. ${ }^{*} p<0.05 ;{ }^{* *} p<0.01$; paired $t$ test $(\boldsymbol{A}-\boldsymbol{D})$ or one-way repeated-measures ANOVA followed by Tukey multiple comparisons test $(\boldsymbol{F}-\boldsymbol{K})$.

did not increase mean firing (Fig. 2C). During the capsaicinevoked response, stroking did not have an effect on the mean firing rate after stroking (cotton: from $1.11 \pm 0.26$ to $1.27 \pm 0.26$ impulses/s; thin brush: from $1.37 \pm 0.36$ to $1.59 \pm 0.60$ impulses/s; thick brush: from $0.89 \pm 0.23$ to $1.34 \pm 0.35$ impulses/s; Fig. $2 I-K)$.

To test whether myelinated fibers mediate post-stroking inhibition of pruritogen-responsive neurons, we used a Toll-like receptor 5 (TLR5)-dependent A-fiber silencing approach: coinjection of a sodium channel blocker, QX-314, and a ligand of TLR5, flagellin (Xu et al., 2015; Pan et al., 2019; Sakai and Akiyama, 2019). Activation of TLR5 with flagellin leads to selective QX-314 entry into A $\beta$ LTMR fibers and subsequent blockade of sodium currents in these fibers. To verify the selectivity of the silencing approach, we performed in vivo single-unit recording from the DRG. A representative $\mathrm{A} \beta$-LTMR fiber was silenced for more than 3 h by coinjection of flagellin (10 pmol) and QX-314 (120 nmol) (Fig. 3A). A lower dose of QX-314 $(24 \mathrm{nmol})$ also silenced a representative A $\beta$-LTMR fiber, with a shorter duration (Fig. $3 B$ ). Coinjection of flagellin (10 pmol) and QX-314 (120 nmol) did not silence either an A $\delta$-LTMR fiber (Fig. 3C) or a C-LTMR fiber (Fig. 3D). Consistent with previous reports (Xu et al., 2015; Pan et al., 2019; Sakai and Akiyama, 2019), coinjection of QX-314 and flagellin into the hindpaw selectively silenced A $\beta$-LTMR fibers in vivo.

Next, following coinjection of QX-314 and flagellin, we performed in vivo single-unit recording from the spinal cord. Ten units were isolated using an i.d. chloroquine search stimulus (Fig. 4). The mean recording depth was $61.8 \pm 27.5 \mu \mathrm{m}$, and histologically identified recording sites were in the superficial dorsal horn 

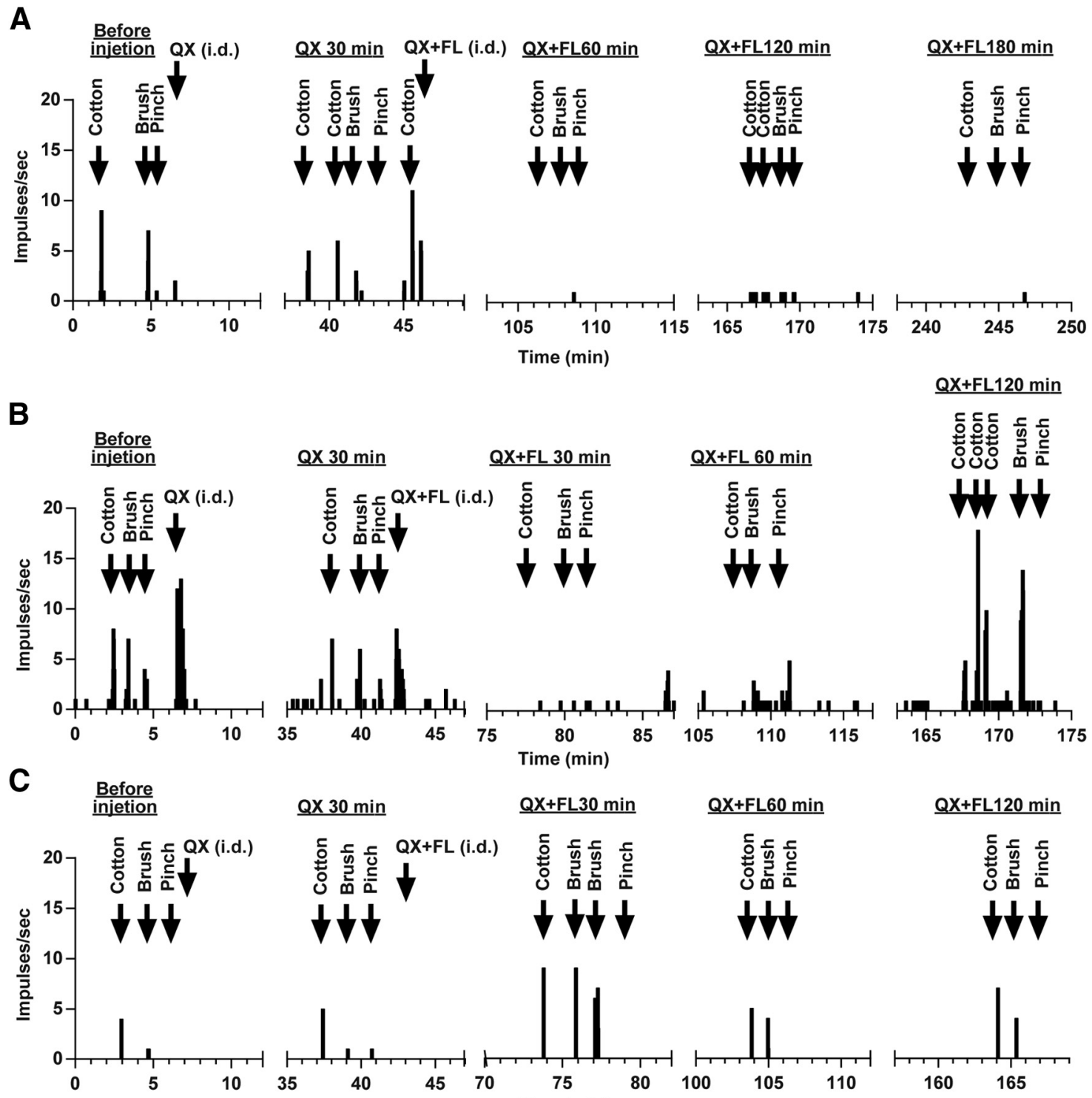

$\underline{Q \times 30 \mathrm{~min}}$

$\underline{Q X+F L 30 ~ m i n ~}$

$\underline{Q X+F L 60 ~ m i n}$

$\underline{Q X+F L 120 ~ m i n ~}$
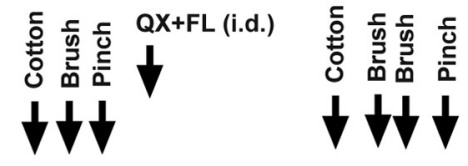

ठํำ
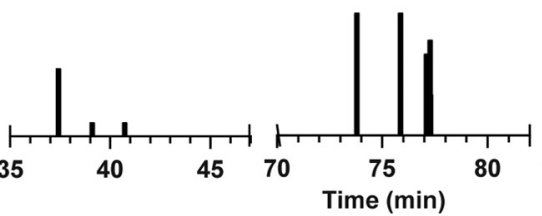

$\downarrow$

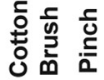

$\downarrow \downarrow \downarrow$

D $\quad$ Before

QX30 min

$\underline{Q X+F L 30 ~ m i n}$
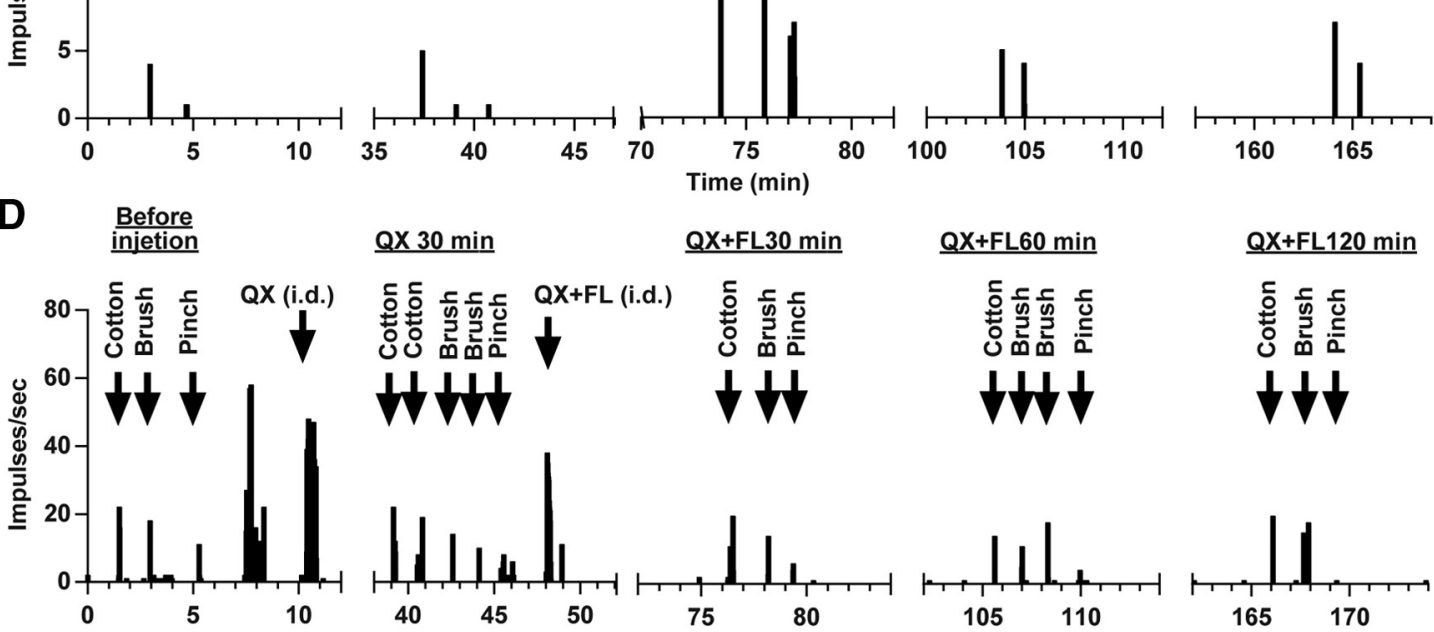

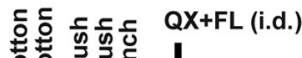

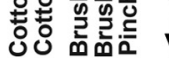

HWt

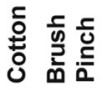

$\downarrow W$

$\underline{Q X+F L 60 \min }$

$\underline{Q X+F L 120 \min }$
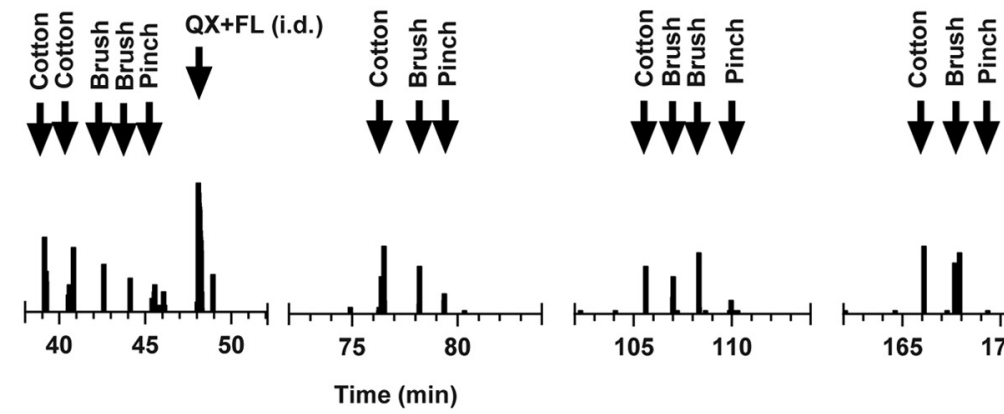

Figure 3. Pharmacological silencing of TLR5 ${ }^{+}$myelinated fibers. Cutaneous LTMRs were recorded using in vivo single-unit electrophysiological recording from the DRGs in C57BL/6J mice. The recorded units were classified as $A \beta-, A \delta$-, and C-LTMR fibers based on their conduction velocities and mechanosensitivities. TLR5 ${ }^{+}$myelinated fibers were silenced by coinjection of QX314 and flagellin to the hindpaw. $\boldsymbol{A}$, Coinjection of QX (QX-314; $120 \mathrm{nmol}$ ) and FL (flagellin; $10 \mathrm{pmol}$ ) abolished the responses of $\mathrm{A} \beta$-LTMR to cotton and thick brush for up to $3 \mathrm{~h}$. $\boldsymbol{B}$, Coinjection of low-dose QX (24 nmol) and FL (10 pmol) abolished the responses of A $\beta$-LTMR to cotton and thick brush for a shorter duration. C, D, Coinjection of QX (120 nmol) and FL $(10 \mathrm{pmol})$ had no effects on the mechanosensitivities of A $\delta$-LTMR ( $(C)$ and $(-$ LTMR $(\boldsymbol{D})$ fibers.

(Fig. 4A, inset). During the chloroquine-evoked response, the mean firing was not significantly changed during stroking with the thick brush (from $1.49 \pm 0.37$ to $1.81 \pm 0.24$ impulses/s). The mean firing rate after thick brush was significantly lower
$(0.75 \pm 0.19$ impulses/s) compared with the mean firing rate before thick brush $\left(1.49 \pm 0.37\right.$ impulses/s, $F_{(1.43,7.16)}=16.00$, $p=0.033$, Tukey's multiple comparison test: $p=0.0034$; Fig. $4 C$ ). Therefore, TLR5-dependent myelinated fiber silencing was not 
sufficient to block stroking-evoked inhibition of pruritogen-responsive neurons.

Optogenetic inhibition of VGLUT3-lineage sensory nerves reduces post-stroking inhibition of chloroquine-responsive neurons

VGLUT3-lineage sensory nerves are thought to mediate innocuous touch, such as stroking the skin (Seal et al., 2009; Abraira and Ginty, 2013; Lou et al., 2013). We next asked whether VGLUT3-lineage sensory nerves mediate poststroking inhibition of pruritogen-responsive spinal neurons. To visualize the innervation of VGLUT3lineage sensory nerves in the skin, we bred Vglut3cre mice with tdTomato reporter mice (Madisen et al., 2010). We then performed immunohistochemistry using skin sections from Vglut3-cre/tdTomato mice. The majority of tdTomato-expressing nerve fibers coexpressed VGLUT3 (Fig. 5A). The tdTomato-expressing nerve fibers $(22.7 \%, 476$ of 2101) represent a small proportion of PGP9.5-positive epidermal nerves (Fig. 5B). Figure $5 C$ shows that tdTomato-expressing nerve fibers also coexpressed NFH, previously reported as a marker for myelinated neurons in touch domes (Lou et al., 2013). A few tdTomato-expressing epidermal nerve fibers coexpressed CGRP, a peptidergic Cfiber marker (6.9\%, 65 of 936; Fig. 5D), or P2X3, a nonpeptidergic C-fiber marker (10.0\%, 67 of 665 ; Fig. $5 E$ ), which is consistent with a previous report (Draxler et al., 2014).

To silence VGLUT3-lineage sensory nerves, we prepared Vglut3-cre/NpHR-EYFP mice. In skin sections from these mice, peripheral EYFPexpressing nerve fibers stained positively for VGLUT3 (Fig. 6A). Using in vivo single-unit recording, we identified 14 chloroquine-responsive dorsal horn units in Vglut3-cre/NpHR-EYFP mice (Fig. 6). Of these, 13 (93\%) responded to capsaicin. None of the units responded to either blue or green light stimuli. Most unit recording sites were located in the superficial dorsal horn at a mean depth of $127.1 \pm 27.0 \mu \mathrm{m}$ below the surface of the lumbar spinal cord. For most units, the location was confirmed by histologic identification of lesion sites (Fig. 6B, inset).

PBS vehicle did not increase mean unit firing (Fig. 6B). As expected, mean firing immediately after chloroquine injection was significantly greater compared with the pre-injection level (Fig. 6C). Likewise, mean unit firing immediately after capsaicin injection was significantly greater compared with the pre-injection level, while PBS-Tween vehicle did not increase mean firing (Fig. 6D,E). Effects of stroking the hindpaw with the thick brush on chloroquine-evoked firing were biphasic in Vglut3-crel NPHR-EYFP mice. Chloroquine-evoked firing was significantly enhanced during stroking (from $0.62 \pm 0.08$ to $6.88 \pm 2.33$ impulses/s; Fig. 6I), followed by a significant reduction in firing post-stroking $\left(0.29 \pm 0.07\right.$ impulses/s, $F_{(1.00,6.01)}=7.44$, $p=0.034$, Tukey's multiple comparison test: $p=0.0020$; Fig. $6 I$ ). Simultaneous green light stimulation during stroking canceled the post-stroking suppression of neuronal firing (from
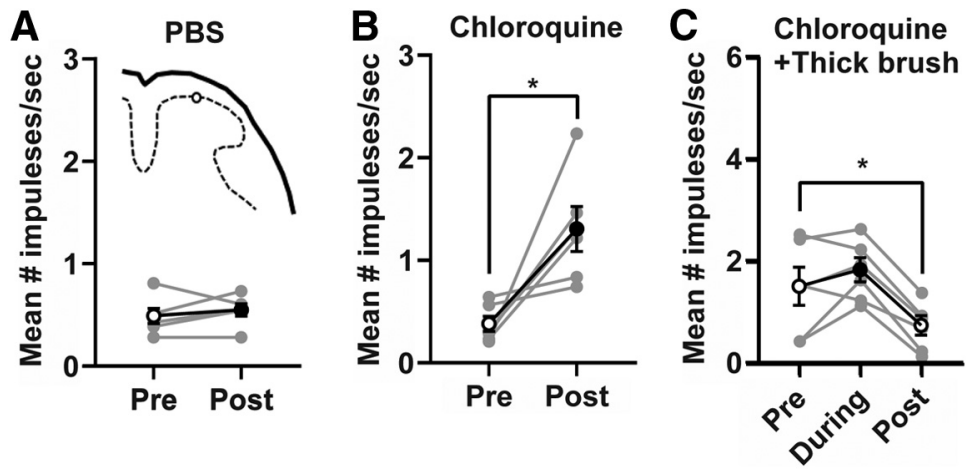

Figure 4. Myelinated fiber silencing was not sufficient to block stroking-evoked inhibition of chloroquine-evoked firing in the spinal cord in $\mathrm{C57BL} / 6 \mathrm{~J}$ mice. TLR5 ${ }^{+}$myelinated fibers were silenced by coinjection of QX-314 $(120 \mathrm{nmol})$ and flagellin $(10 \mathrm{pmol})$ to the hindpaw. $\boldsymbol{A}$, Average firing of units recorded from the superficial dorsal horn of spinal cord before (pre) and after (post) i.d. PBS. Inset, Recording sites. $\boldsymbol{B}$, Same as in $\boldsymbol{A}$, for i.d. chloroquine. $\boldsymbol{C}$, Individual (gray lines) and mean (black line) responses of superficial dorsal horn units following i.d. chloroquine, before (pre), during, and after (post) stroking with the thick brush. Data are mean \pm SEM. ${ }^{*} p<0.05$; paired $t$ test $(\boldsymbol{A}, \boldsymbol{B})$ or one-way repeated measures ANOVA followed by Tukey multiple comparisons test (C).
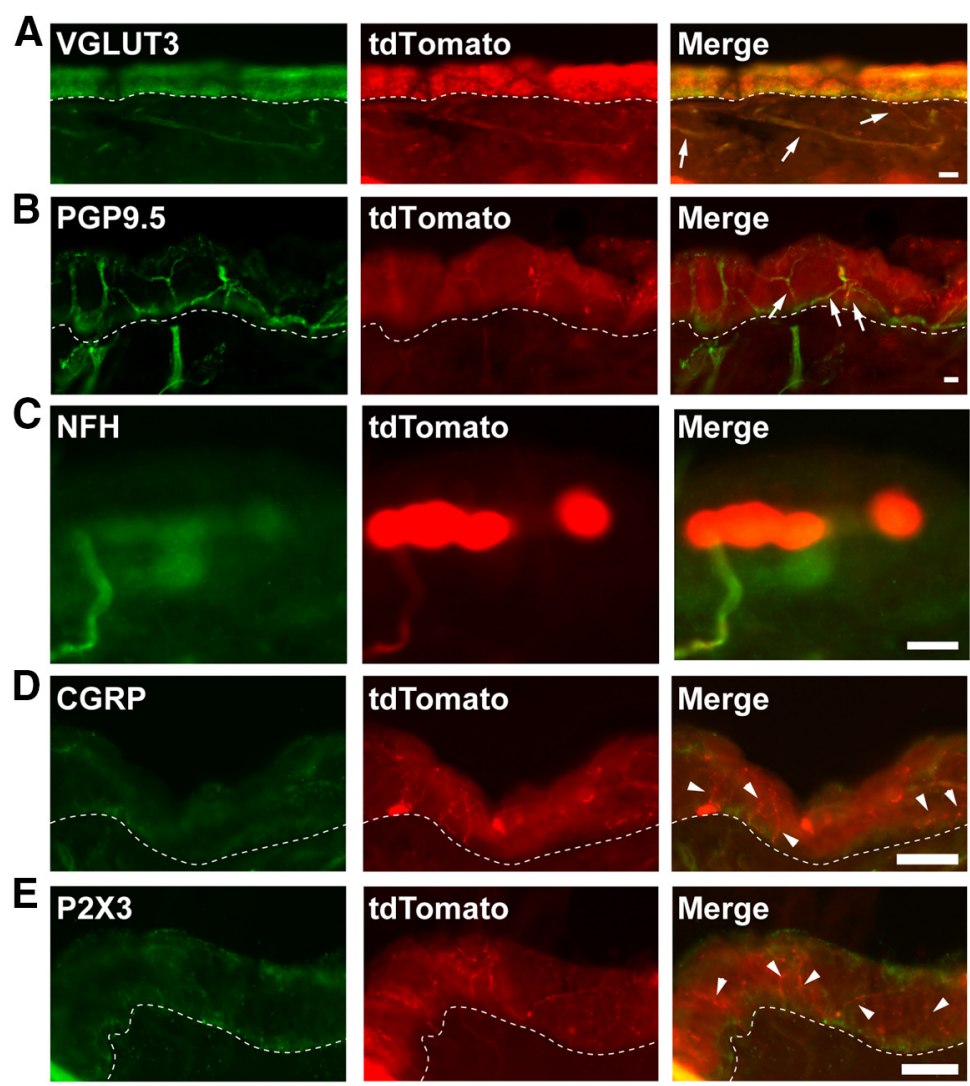

Figure 5. Innervation of VGLUT3-lineage sensory nerves in the skin. Skin sections from Vglut3-cre/TdTomato mice were immunostained with antibodies for VGLUT3 (green; $\boldsymbol{A}$ ), PGP9.5 (B), NFH (C), CGRP (D), or P2X3 (E). Arrows indicate double-positive nerves. Arrowheads indicate tdTomato single-positive nerves. Scale bars, $10 \mu \mathrm{m}$.

$0.75 \pm 0.11$ to $0.98 \pm 0.20$ impulses/s; Fig. $6 G, H)$ without affecting the enhancing effect of stroking with the thick brush. Simultaneous blue light (control) stimulation during stroking had no effect on post-stroking firing (from $0.68 \pm 0.17$ to $0.3 \pm 0.07$ impulses/s; Fig. $6 F, H$ ), These data suggest that VGLUT3-lineage sensory nerves mediate stroking-evoked inhibition of pruritogen-responsive neurons.

Stroking the hindpaw with the thick brush did not affect post-stroking activity elicited by capsaicin in Vglut3-cre/NpHR- 


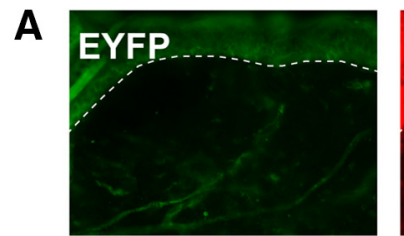

B

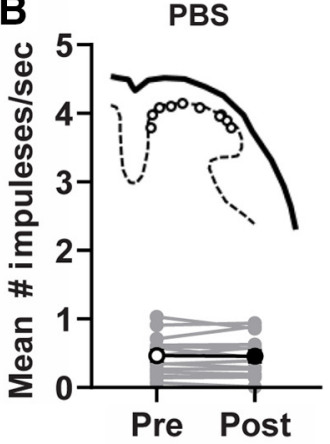

$\mathbf{F}$
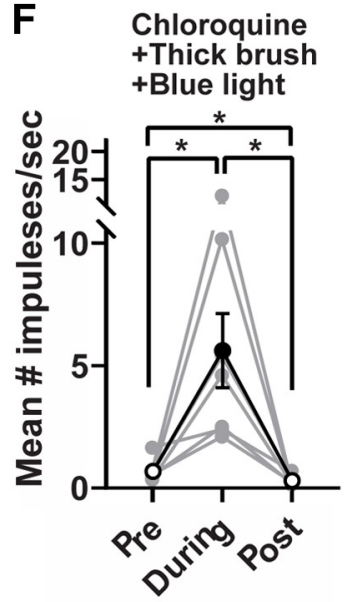

I
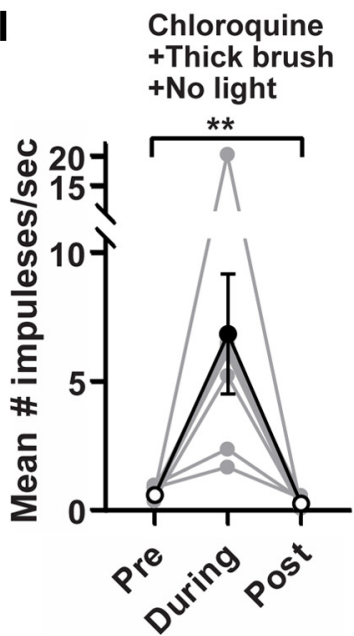
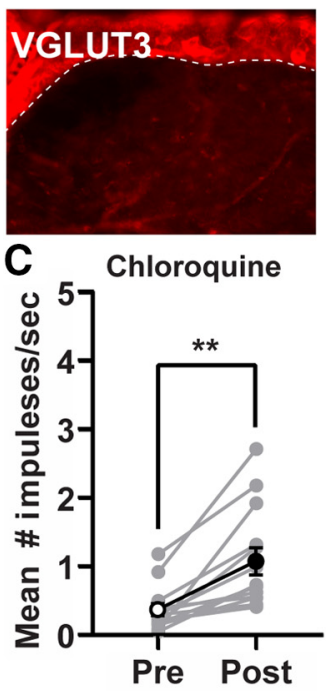

G

Chloroquine

+Thick brush

+Green light

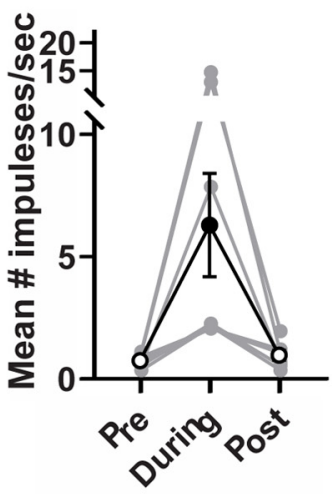

J

Capsaicin +Thick brush +Blue light

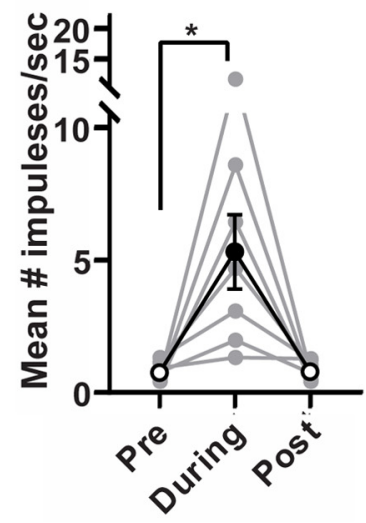

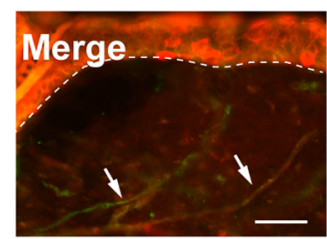
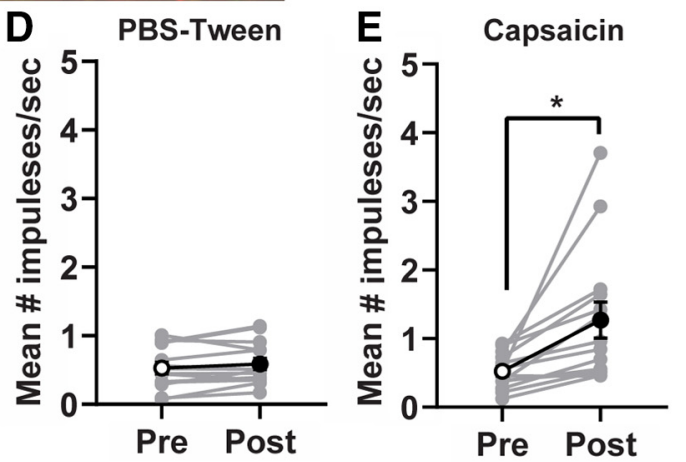

H

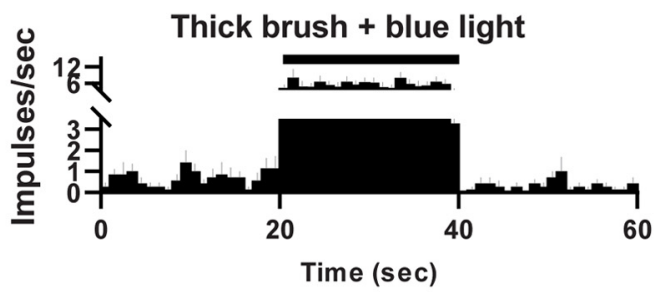

Thick brush + green light

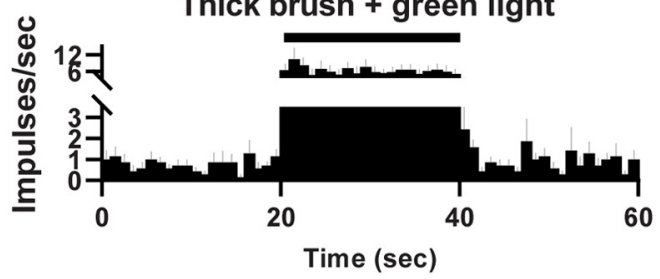

K

Capsaicin +Thick brush +Green light

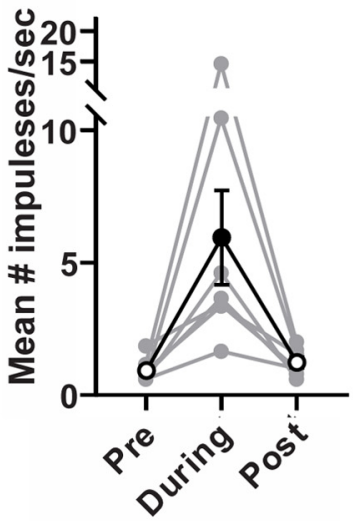

L

Capsaicin +Thick brush

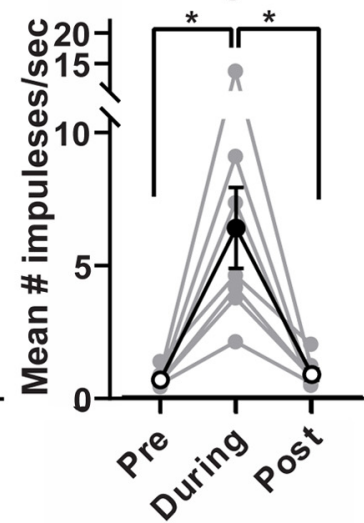
+No light

Figure 6. Optogenetic inhibition of VGLUT3-lineage sensory nerves blocked post-stroking inhibition of chloroquine-evoked firing in the spinal cord of Vglut3-cre/NpHR-EYFP mice. A, Skin sections from Vglut3-cre/NpHR-EYFP mice were immunostained for VGLUT3 (red) to show expression of halorhodopsin in VGLUT3 ${ }^{+}$sensory nerves of the skin. Arrows indicate double-positive nerves. Scale bars, $10 \mu \mathrm{m}$. B, Average firing of units recorded from the superficial dorsal horn of spinal cord before (pre) and after (post) i.d. PBS. Inset, Spinal recording sites. $\boldsymbol{C}$, Same as in $\boldsymbol{B}$, for i.d. chloroquine injection. $\boldsymbol{D}$, Same as in $\boldsymbol{B}$, for i.d. PBS-Tween injection (vehicle control for capsaicin injection). $\boldsymbol{E}$, Same as in $\boldsymbol{B}$, for i.d. capsaicin injection. $\boldsymbol{F}$, Individual (gray lines) and mean (black line) responses of superficial dorsal horn units following i.d. chloroquine before (pre), during, and after (post) stroking with the thick brush and simultaneous blue light exposure. $\boldsymbol{G}$, Same as in $\boldsymbol{F}$, for green light exposure. $\boldsymbol{H}$, Averaged peristimulus time histograms (bins: $1 \mathrm{~s}$ ) of activity following i.d. chloroquine before, during, and after stroking with the thick brush and simultaneous blue or green light exposure. Horizontal lines indicate duration of stroking and light exposure. $\boldsymbol{I}$, Same as in $\boldsymbol{F}$, for no light exposure. J, Individual (gray lines) and mean (black line) responses of superficial dorsal horn units following i.d. capsaicin before (pre), during, and after (post) stroking with the thick brush and simultaneous blue light exposure. $\boldsymbol{K}$, Same as in $\boldsymbol{J}$, for green light. $\boldsymbol{L}$, Same as in $\boldsymbol{J}$, for no light. Data are mean \pm SEM. ${ }^{*} p<0.05$; ${ }^{* *} p<0.01$; paired $t$ test $(\boldsymbol{B}-\boldsymbol{E})$ or one-way repeated measures ANOVA followed by Tukey multiple comparisons test $(\boldsymbol{F}, \boldsymbol{G}, \mathbf{I}-\boldsymbol{L})$. 
A
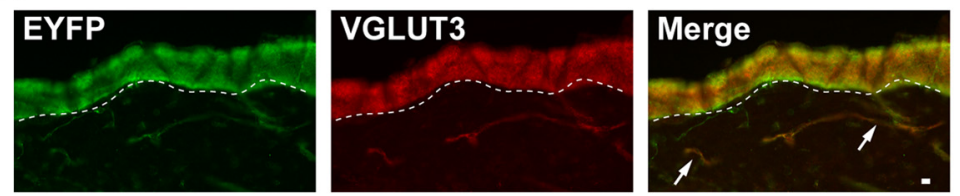

B

Blue light, $20 \mathrm{~Hz}$

Green light, $20 \mathrm{~Hz}$

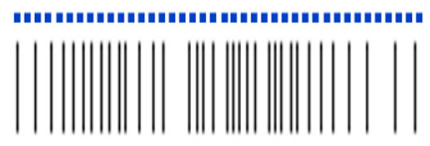

C
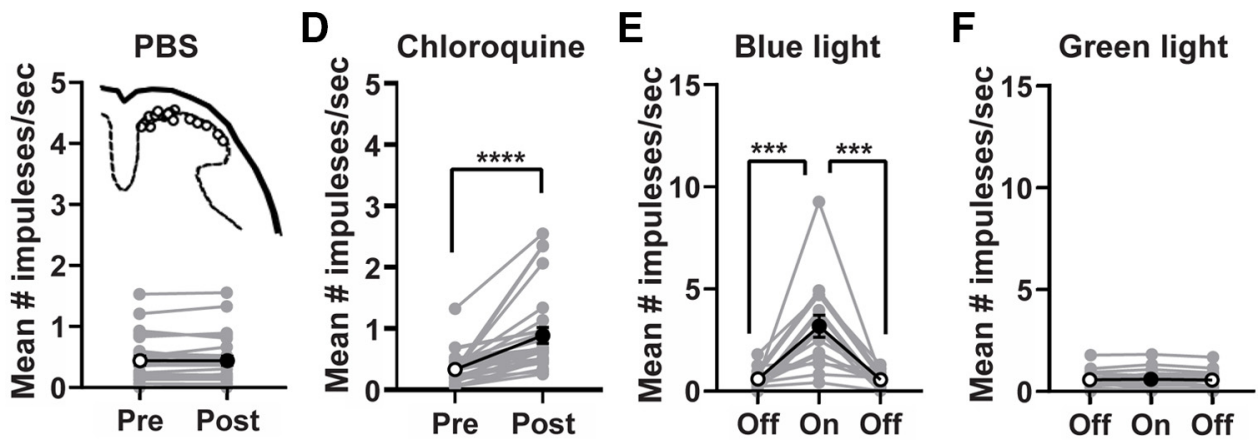

\section{G

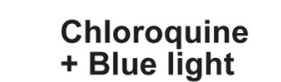

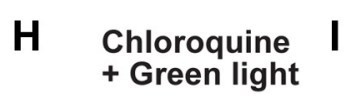
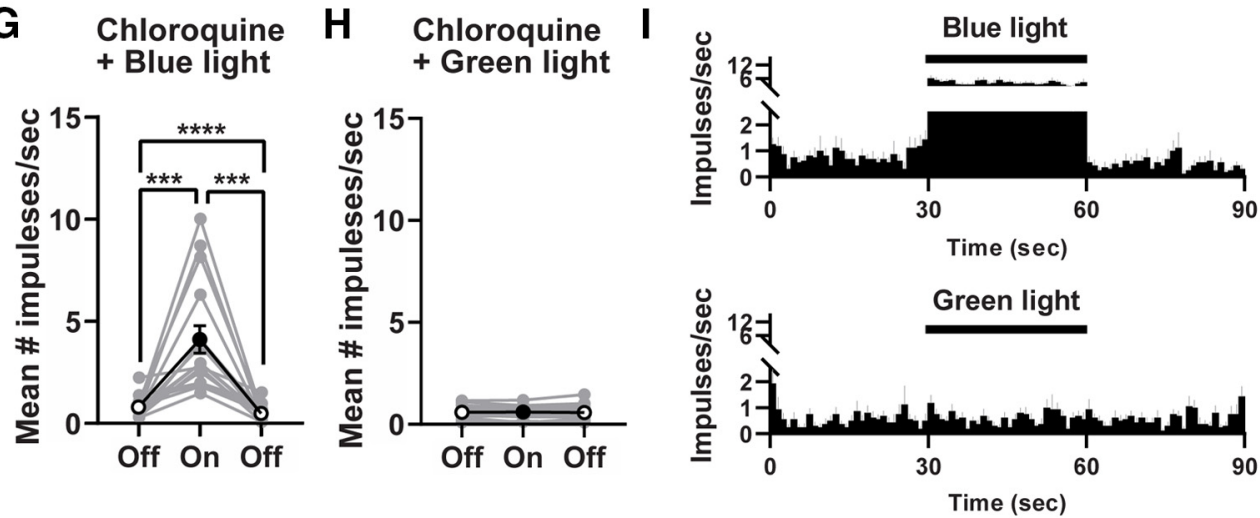

Figure 7. Optogenetic stimulation of VGLUT3-lineage sensory nerves inhibited chloroquine-evoked firing in the spinal cord in Vglut3-cre/ChR2-EYFP mice. A, Skin sections from Vglut3-cre/ ChR2-EYFP mice were immunostained for VGLUT3 (red). Arrows indicate double-positive nerves. Scale bars, $10 \mu \mathrm{m}$. B, Firing responses of a C-LTMR fiber to blue or green light pulses (20 Hz). A C-LTMR unit was recorded from the DRGs in Vglut3-cre/ChR2-EYFP mice. C, Average firing of units recorded from the superficial dorsal horn of spinal cord before (pre) and after (post) i.d. PBS. Inset, Recording sites. $\boldsymbol{D}$, Same as in $\boldsymbol{C}$, for chloroquine injection. $\boldsymbol{E}$, Individual (gray lines) and mean (black line) responses of superficial dorsal horn units before (pre), during, and after (post) blue light stimulation. $\boldsymbol{F}$, Same as in $\boldsymbol{E}$, for green light stimulation. $\boldsymbol{G}$, Individual (gray lines) and mean (black line) responses of superficial dorsal horn units following i.d. chloroquine, before (pre), during, and after (post) blue light stimulation. $\boldsymbol{H}$, Same as in $\mathbf{G}$, for green light stimulation. $\boldsymbol{I}$, Averaged peristimulus time histogram (bins: $1 \mathrm{~s}$ ) of activity following i.d. chloroquine before, during, and after blue or green light stimulation. Horizontal lines indicate duration of light stimulation. Data are mean $\pm \mathrm{SEM}$. ${ }^{* * *} p<0.001 ;{ }^{* * *} p<0.0001$; paired $t$ test $(\boldsymbol{C}, \boldsymbol{D})$ or one-way repeated-measures ANOVA followed by Tukey multiple comparisons test $(\boldsymbol{E}-\boldsymbol{H})$.

EYFP mice (from $0.7 \pm 0.12$ to $0.9 \pm 0.21$ impulses/s; Fig. $6 L$ ). Neither simultaneous blue nor green light stimulation during stroking had any significant effects on post-stroking firing (green: from $0.93 \pm 0.17$ to $1.23 \pm 0.20$ impulses/s; blue: from $0.78 \pm 0.13$ to $0.81 \pm 0.13$ impulses/s; Fig. $6 \mathrm{~J}, \mathrm{~K})$.

Optogenetic stimulation of VGLUT3-lineage sensory nerves inhibits responses of spinal neurons to itch stimuli

To investigate whether optogenetic stimulation of VGLUT3-lineage sensory nerves inhibits ongoing activity elicited by pruritogens in the spinal cord, we prepared Vglut3-cre/ChR2-EYFP mice. In skin sections from Vglut3-cre/ChR2-EYFP mice, peripheral EYFPexpressing nerve fibers stained positively for VGLUT3 (Fig. 7A). In vivo single-unit recording from the DRG confirmed that blue light delivered to the hindpaw increased the firing rate of a C-LTMR fiber in Vglut3-cre/ChR2-EYFP mouse (Fig. 7B, left). The same unit did not respond to green light (Fig. $7 B$, right). Using in vivo singleunit recording from the lumbar spinal cord, we isolated 25 chloroquine-responsive units in the superficial dorsal horn. Of these units, $62.5 \%$ (10 of 16) responded to histamine injection to the hindpaw (Fig. $8 A$ ), 68.8\% (11 of 16) responded to serotonin injection (Fig. $8 D$ ), and $100 \%$ (6 of 6) responded to capsaicin injection. While none of the chloroquine-responsive units responded to green light stimulus, $100 \%$ ( 25 of 25 ) responded to blue light stimulus. All unit recording sites were located in the superficial dorsal horn at a mean depth of $109.5 \pm 19.3 \mu \mathrm{m}$ below the surface of the lumbar spinal cord. For most units, the location was confirmed by histologic identification of lesion sites (Fig. $7 C$, inset).

As in C57BL/6 mice, mean firing immediately after chloroquine or after capsaicin injection was significantly greater 
A

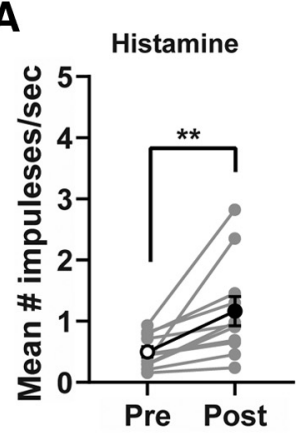

D
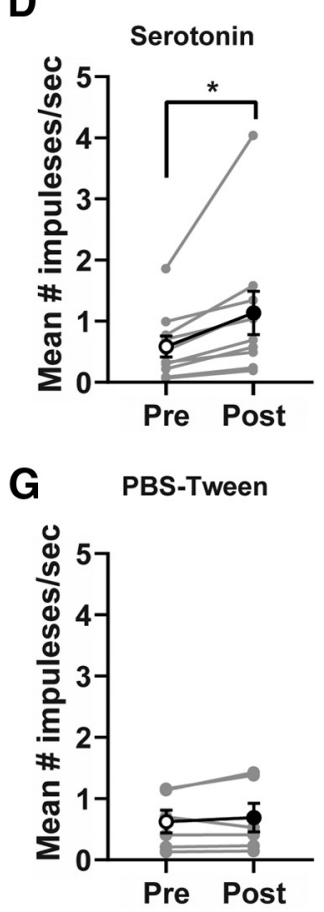

B

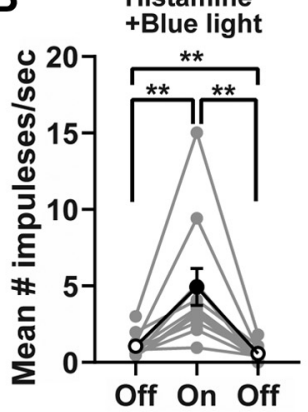

E
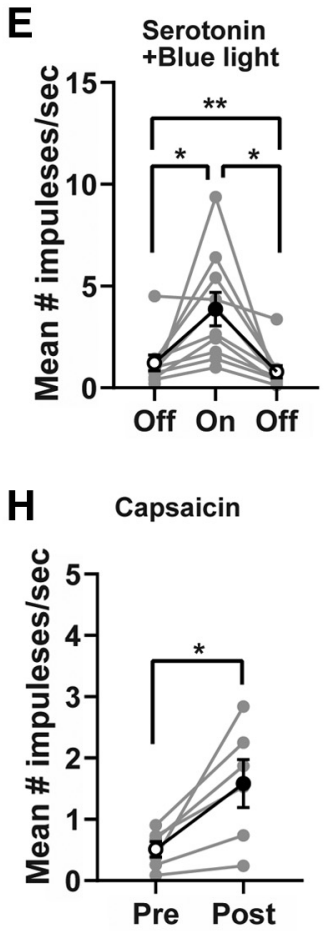
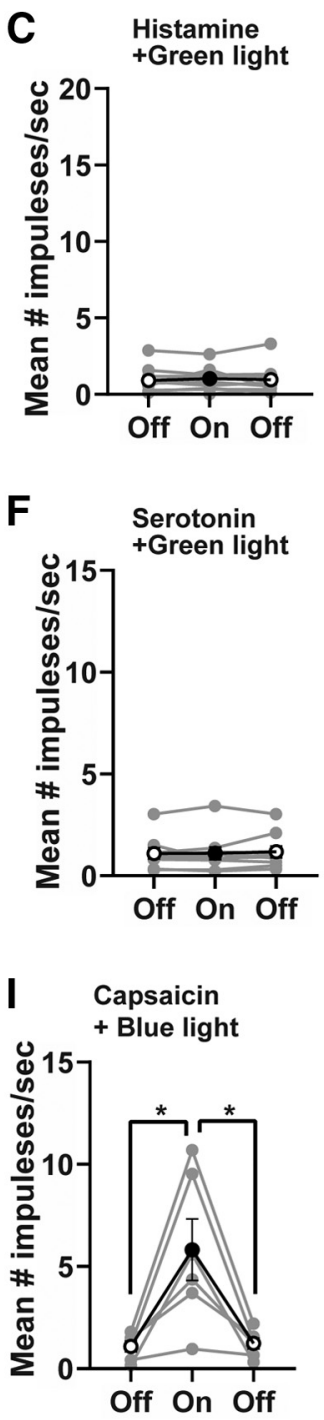

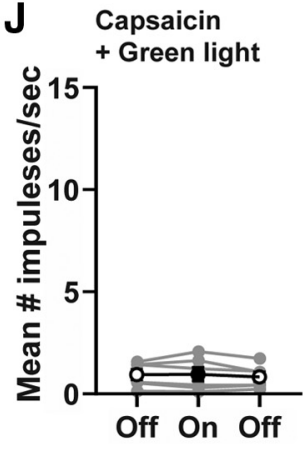

Figure 8. Optogenetic stimulation of VGLUT3-lineage sensory nerves inhibited histamine- or serotonin-evoked firing in the spinal cord in Vglut3-cre/ChR2-EYFP mice. A, Average firing of units recorded from the superficial dorsal horn of spinal cord before (pre) and after (post) i.d. histamine. $\boldsymbol{B}$, Individual (gray lines) and mean (black line) responses of superficial dorsal horn units following i.d. histamine, before (pre), during, and after (post) blue light stimulation. $\boldsymbol{C}$, Same as in $\boldsymbol{B}$, for green light stimulation. $\boldsymbol{D}$, Same as in $\boldsymbol{A}$, for serotonin. $\boldsymbol{E}$, Same as in $\boldsymbol{B}$, for serotonin. $\boldsymbol{F}$, Same as in $\boldsymbol{C}$, for serotonin. $\boldsymbol{G}$, Same as in $\boldsymbol{A}$, for PBS-Tween injection (vehicle control for capsaicin injection). $\boldsymbol{H}$, Same as in $\boldsymbol{A}$, for capsaicin. $\boldsymbol{I}$, Same as in $\boldsymbol{B}$, for capsaicin. $\boldsymbol{J}$, Same as in $\boldsymbol{C}$, for capsaicin. Data are mean \pm SEM. ${ }^{*} p<0.05 ;{ }^{* *} p<0.01$, paired $t$ test $(\boldsymbol{A}, \boldsymbol{D}, \boldsymbol{G}, \boldsymbol{H})$ or one-way repeated-measures ANOVA followed by Tukey multiple comparisons test $(\boldsymbol{B}, \boldsymbol{C}, \boldsymbol{E}, \boldsymbol{F}, \boldsymbol{I}, \boldsymbol{J})$.

compared with the pre-injection level, while PBS or PBS-Tween vehicle did not increase mean firing (Figs. $7 C, D, 8 G, H$ ). During the chloroquine-evoked response, firing was enhanced during blue light stimulus (from $0.8 \pm 0.12$ to $4.11 \pm 0.67$ impulses/s), followed by a significant reduction in firing after blue light stimulus $\left(0.49 \pm 0.09 \mathrm{impulses} / \mathrm{s}, F_{(1.00,15.06)}=25.88, p=0.0001\right.$, Tukey's multiple comparison test: $p=0.00001$; Fig. $7 G, I)$. Similarly, during the histamine- or serotonin-evoked responses, firing after blue light stimulus was significantly reduced compared with firing before blue light stimulus (histamine: before: $1.06 \pm 0.24 \mathrm{impulses} / \mathrm{s}$, after: $0.57 \pm 0.15 \mathrm{impulses} / \mathrm{s}$, $F_{(1.01,0.06)}=15.07, p=0.0030$, Tukey's multiple comparison test: $p=0.0011$; serotonin; before: $1.23 \pm 0.39$ impulses $/ \mathrm{s}$, after: $0.80 \pm 0.30 \mathrm{impulses} / \mathrm{s}, F_{(1.01,9.11)}=10.7, p=0.0094$, Tukey's multiple comparison test: $p=0.0027$; Fig. $8 B, E$ ).

In contrast, during the capsaicin-evoked response, the blue light stimulus did not significantly affect firing after blue light stimulus (before: $1.08 \pm 0.26$ impulses/s; after $1.23 \pm 0.27$ impulses/s; Fig. 8I). The green light (control) stimulus did not significantly affect ongoing activity elicited by any tested reagents (Figs. $7 H, I, 8 C, F, J)$. Therefore, optogenetic stimulation of VGLUT3-lineage sensory nerves was sufficient to inhibit firing elicited by pruritogens in the spinal cord in a state-dependent manner.

Recent studies revealed that there are two distinct subsets of spinal itch inhibitory interneurons, which regulate itch via neuropeptide Y receptor Y1 (NPY1R) or $\kappa$ opioid receptor (KOR) (Ross et al., 2010; Kardon et al., 2014; Bourane et al., 2015; Acton et al., 2019). To investigate which subsets of inhibitory interneurons are involved in inhibition of itch by VGLUT3-lineage sensory nerves, we applied either nor-binaltorphimine dihydrochloride, a KOR antagonist, or BMS193885, an NPY1R antagonist, to the spinal cord. To determine the duration of the inhibitory effects of antagonists, we identified mechano-sensitive spinal neurons and tested their responses to mechanostimuli before and after treatment with the antagonists. The responses to pinch were increased at $2 \mathrm{~min}$ after treatment with the antagonists, followed by returning to baseline (Fig. 9A,B). Therefore, 
the effects of antagonists were tested at 2 min after application in the following experiments.

We tested nine chloroquine-responsive units with the two antagonists. None of the units exhibited an increased firing rate following the application of nor-binaltorphimine dihydrochloride or BMS193885 alone. During the chloroquine-evoked response, firing was significantly reduced after blue light stimulus (from $0.75 \pm 0.12$ to $0.40 \pm 0.09$ impulses/s, $\quad F_{(1.01,8.09)}=14.53, \quad p=0.005$, Tukey's multiple comparison test: $p=0.0005$; Fig. 9C). Application of nor-binaltorphimine dihydrochloride during the blue light stimulus canceled the poststimulus suppression of neuronal firing (from $1.27 \pm 0.25$ to $1.26 \pm 0.22$ impulses/s; Fig. 9D). Application of BMS193885 during the blue light stimulus had no effect on post-stimulus firing (from $1.29 \pm 0.28$ to $0.49 \pm 0.11$ impulses/s; Fig. 9E). Neither antagonist had an effect on blue lightevoked firing after PBS injection (Fig. 9F-H). These data suggest that KOR-expressing spinal interneurons mediate the itch-inhibitory effects of VGLUT3lineage nerves.

\section{Optogenetic stimulation of VGLUT3-lineage sensory nerves inhibits itch-related behavior, but not pain-related behavior}

To investigate whether optogenetic stimulation of VGLUT3-lineage sensory nerves affects itchrelated behavior, we used Vglut3-cre/ChR2-EYFP mice and videotaped their behaviors following i.d. injection of the pruritogens histamine, serotonin, and chloroquine with and without simultaneous blue or green light stimulation. Blue light stimulation of peripheral VGLUT3-lineage sensory nerves significantly reduced the total scratching response to each pruritogen (histamine: $F_{(1.62,12.92)}=9.719$, $p=0.0039$, Tukey's multiple comparison test: $p=$ 0.011 ; serotonin: $F_{(1.22,10.96)}=6.62, p=0.022$, Tukey's multiple comparison test: $p=0.0069$; chloroquine: $F_{(1.67,15.05)}=10.7, p=0.0017$, Tukey's multiple comparison test: $p=0.012$; Fig. $10 A-C$ ). In contrast, green light (control) stimulation did not have any significant effects on the total scratching response. Neither blue nor green light affected spontaneous scratching in Vglut3-cre/ChR2-EYFP mice (Fig. 10D)

Finally, to assess whether optogenetic stimulation of peripheral VGLUT3-lineage sensory nerves affects pain sensation, we used the Hargreaves test and von Frey filament test in Vglut3cre/ChR2-EYFP mice. Neither blue light nor green light stimulation had any effect on thermal or mechanical pain thresholds (Fig. 10E,F).

Collectively, these data demonstrate that optogenetic stimulation of VGLUT3-lineage sensory nerves is capable of inhibiting itch-related behavior without affecting pain-related behaviors.

\section{Discussion}

Innocuous mechanical stimuli (e.g., rubbing or stroking the skin) are thought to relieve itch through the activation of LTMRs. Here we show that stroking the skin inhibits the responses of spinal neurons to pruritogens via VGLUT3-lineage $(-H)$.
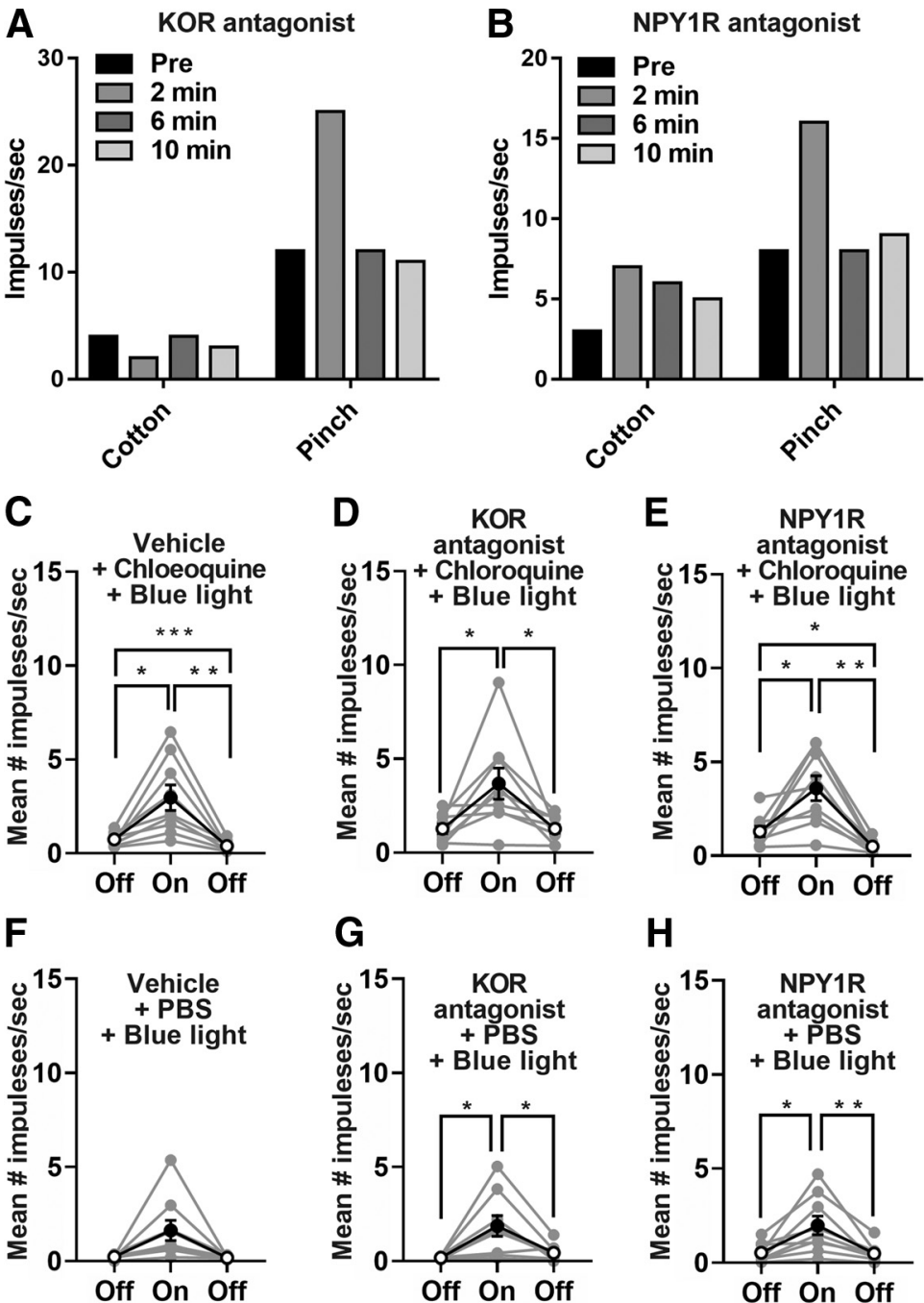

Figure 9. A KOR antagonist canceled the itch inhibitory effects of optogenetic stimulation of VGLUT3-lineage sensory nerves in Vglut3-cre/ChR2-EYFP mice. $\boldsymbol{A}$, Responses of a superficial dorsal unit to cotton and pinch before and after superfusion of the KOR antagonist nor-binaltorphimine dihydrochloride. $\boldsymbol{B}$, Same as in $\boldsymbol{A}$, for NPY1R tagonist BMS193885. C, Individual (gray lines) and mean (black line) responses of superficial dorsal horn units ing stimulation. $\boldsymbol{D}$, Same as in $\boldsymbol{C}$, for KOR antagonist. $\boldsymbol{E}$, Same as in $\boldsymbol{C}$, for NPY1R antagonist. $\boldsymbol{F}$, Same as for PBS. $\boldsymbol{G}$, Same as in $\boldsymbol{D}$, for PBS. $\boldsymbol{H}$, Same as in $\boldsymbol{E}$, for PBS. Data are mean \pm SEM. ${ }^{*} p<0.05$ ${ }^{*} p<0.01 ;{ }^{* *} p<0.001$; one-way repeated-measures ANOVA followed by Tukey multiple comparisons test

sensory nerves. Moreover, optogenetic stimulation of VGLUT3lineage sensory nerves inhibits itch-related behavior without affecting pain-related behaviors.

A cotton swab and two different sizes of brushes were used to stroke the skin. Interestingly, only the thick brush was sufficient to inhibit post-stroking pruritogen-evoked firing of spinal neurons. The strength of stroking with the thick brush was 24$35 \mathrm{mN}$, which is higher than that with cotton swab $(<1 \mathrm{mN})$, implying that the strength of stroking is an important factor for itch inhibition. In line with this, weak stroking $(1-2 \mathrm{mN})$ of the hindpaw skin did not show any effects on spontaneous firing in a mouse model of dry skin. In addition to strength, the width of the device is likely a key factor. While the stroking forces of the two brushes were comparable, the thick brush was $6 \mathrm{~mm}$ wide (stroked area $36 \mathrm{~mm}^{2}$ ) and the thin brush was $2.6 \mathrm{~mm}$ wide (stroked area $15 \mathrm{~mm}^{2}$ ). The receptive fields of rapidly adapting Type 1 LTMRs, slowly adapting Type 1 LTMRs, and C-LTMRs 
A
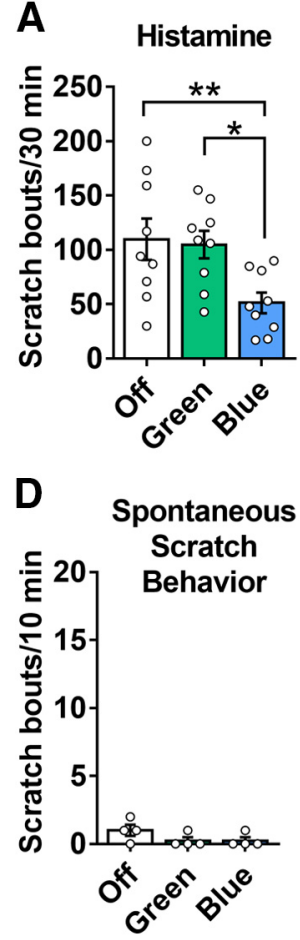
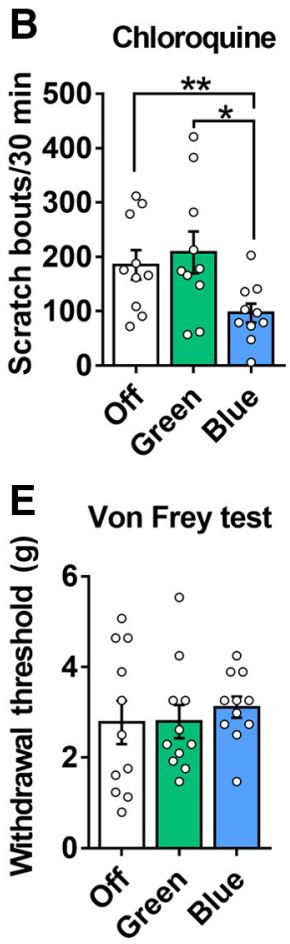
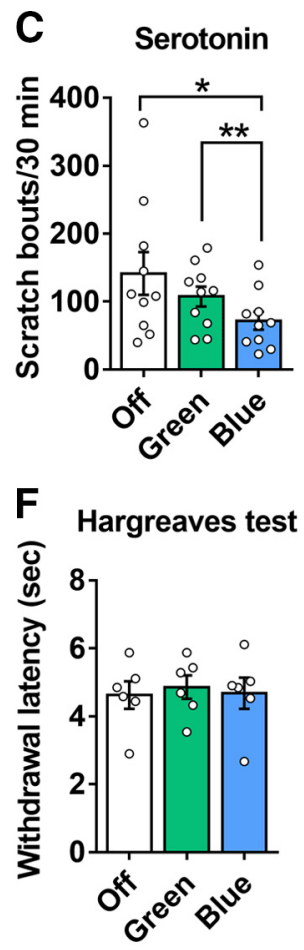

Figure 10. Optogenetic stimulation of VGLUT3-lineage sensory nerves inhibited itch-related behavior without affecting pain-related behaviors in Vglut3-cre/ChR2-EYFP mice. $\boldsymbol{A}$, Number of scratch bouts after i.d. injection of histamine ( $n=9$ /group). $\boldsymbol{B}$, Same as in $\boldsymbol{A}$, for i.d. chloroquine ( $n=10 /$ group). $\boldsymbol{C}$, Same as in $\boldsymbol{A}$, for i.d. serotonin ( $n=10$ /group). $\boldsymbol{D}$, Number of spontaneously occurring scratch bouts $(n=4 /$ group). $\boldsymbol{E}$, Paw withdrawal threshold to von Frey filament ( $n=11 /$ group). $\boldsymbol{F}$, Paw withdrawal latency in the Hargreaves test ( $n=6 /$ group). All experiments were performed under no light, green light, and blue light conditions. Data are mean \pm SEM. ${ }^{*} p<0.05$; ${ }^{* *} p<0.01$; one-way repeated-measures ANOVA followed by Tukey multiple comparisons test.

are 22,9 , and $0.2-0.4 \mathrm{~mm}^{2}$, respectively (Li et al., 2011; Roudaut et al., 2012). The number of activated LTMRs during stroking may be important for post-stroking inhibition of itch.

Stroking the skin activates various types of LTMRs (Abraira and Ginty, 2013; Bai et al., 2015). The velocity of stroking is one of the factors that determines which LTMRs respond. While C-LTMRs respond optimally to skin stroking in the range of $1-10 \mathrm{~cm} / \mathrm{s}$, A-LTMRs show stronger responses in proportion to higher velocities (Loken et al., 2009). In the present study, the stroking velocity was $\sim 1.2 \mathrm{~cm} / \mathrm{s}$. This could likely activate both C- and A-LTMRs. Stroking-evoked inhibition of itch was prevented by optogenetic silencing of VGLUT3-lineage nerves, suggesting that VGLUT3-lineage C-LTMRs or A $\beta$-LTMRs are involved in itch inhibition. Given that selective inhibition of TLR5 $^{+}$A-fibers did not affect stroking-evoked inhibition of itch, VGLUT3-lineage C-LTMRs might play a major role in inhibition of itch by stroking.

There are at least two distinct subsets of itch inhibitory spinal interneurons, including $\mathrm{NPY}^{+}$and Bhlhb5 ${ }^{+}$interneurons (Ross et al., 2010; Kardon et al., 2014; Bourane et al., 2015; Pan et al., 2019). $\mathrm{NPY}^{+}$inhibitory interneurons receive monosynaptic or polysynaptic input from $\mathrm{A} \beta$-, $\mathrm{A} \delta$-, and C-LTMRs (Bourane et al., 2015), suggesting that stroking the skin activates $\mathrm{NPY}^{+}$inhibitory interneurons. A recent study showed that $\mathrm{NPY}^{+}$inhibitory interneurons regulate mechanical itch through NPY1R (Acton et al., 2019). In this study, the NPY1R antagonist BMS193885 failed to prevent inhibition of itch by VGLUT3-lineage nerve stimulation. Post-stroking itch inhibition may be mediated by interneurons other than $\mathrm{NPY}^{+}$inhibitory interneurons. Another subset of itch inhibitory interneurons has been shown to suppress chemical itch via KOR (Kardon et al., 2014). In the present study, the KOR antagonist nor-binaltorphimine dihydrochloride reversed the inhibitory effects of VGLUT3-lineage nerve stimulation. This result suggests that $\mathrm{Bhlhb} 5^{+}$inhibitory spinal interneurons may mediate post-stroking itch inhibition. In line with this, C-LTMR fibers establish direct synaptic contact with GABAergic interneurons to suppress C-fibers in the superficial spinal cord (Lu and Perl, 2003; Kambrun et al., 2018). Whether C-LTMR fibers directly send signals to Bhlhb5 ${ }^{+}$GABAergic interneurons is still unknown.

Previous studies reported that scratching inhibits itch-signaling neurons in the spinal cord in a state-dependent manner (Davidson et al., 2009; Akiyama et al., 2012; Nishida et al., 2013). In primate and rodent pruritogen-responsive spinal neurons, scratching reduces pruritogeninduced firing, but not capsaicin-induced firing. Likewise, in the present study, stroking or stimulation of VGLUT3-lineage nerves reduced the responses of spinal neurons to pruritogens, but not capsaicin. These findings suggest that scratching, stroking, and stimulation of VGLUT3lineage nerves may engage similar inhibitory mechanisms during itch, but not pain.

Stroking the skin at the speeds of $0.1-10 \mathrm{~cm} / \mathrm{s}$ (the optimal speed for C-LTMRs) is known to reduce pain (Shaikh et al., 2015; Habig et al., 2017; Liljencrantz et al., 2017; Gursul et al., 2018). A previous study showed that stroking the skin with sandpaper, an unpleasant stimulus, enhanced underlying muscle pain, whereas stroking with velvet, a pleasurable stimulus, relieved pain (Shaikh et al., 2015). This finding suggests that stroking-evoked analgesia is a type of pleasure-related analgesia, which is elicited by any pleasant stimuli (e.g., pleasant odors, positive images, pleasurable music, and sweet food) (Leknes and Tracey, 2008). Pleasure-related analgesia is expected to be mediated via cortical mechanisms. In contrast to pain, itch relief and pleasant sensation are likely mediated by different mechanisms. Scratch-evoked relief of itch is independent of pleasant sensation and mediated by spinal mechanisms (Davidson et al., 2009; Mochizuki et al., 2017). Likewise, our data suggest that the spinal cord plays an important role in stroking-evoked inhibition of itch.

The gate control theory proposes that touch relieves pain in the spinal cord (Melzack and Wall, 1965). Electrophysiological studies imply that multireceptive neurons in the deep dorsal horn are responsible for pain relief by touch (Hillman and Wall, 1969; Salter and Henry, 1990; Le Bars, 2002). These neurons have segmental receptive fields, which are characterized by an excitatory receptive field surrounded by an inhibitory receptive field. The excitatory receptive field is activated by both innocuous and noxious stimuli, whereas the inhibitory receptive field is activated by innocuous stimuli. The activation of inhibitory receptive field can reduce the firing of multireceptive neurons to nociceptive stimuli. The pruriceptive neurons recorded in the present study are not likely to be multireceptive neurons because they were recorded from the superficial dorsal horn and not the deep dorsal horn. In line with this, stroking the skin did not inhibit the firing of spinal neurons during responses to capsaicin in the current study. 
In our recordings of dorsal horn neurons, stroking with the thick brush markedly increased the firing rate of chloroquineresponsive neurons during chloroquine exposure. A similar phenomenon has been observed for scratching. Most pruritogen-sensitive spinal neurons exhibit an increase in firing rate during scratching within the receptive field (Davidson et al., 2009; Akiyama et al., 2012; Nishida et al., 2013). Previous studies showed that most pruritogen-sensitive spinal neurons are a subpopulation of algogen-sensitive spinal neurons (Akiyama et al., 2009, 2014a; Davidson et al., 2012; Cevikbas et al., 2014). Based on these findings, it has been postulated that pruritogens activate pruritogen- and algogen-sensitive spinal neurons to elicit itch, whereas algogens activate a wider population of algogen-sensitive spinal neurons to elicit pain (Akiyama et al., 2009, 2014a; $\mathrm{Ma}, 2010)$. Therefore, while scratching does excite pruritogensensitive neurons, the overall sensation elicited by scratching is pain and not itch. A similar idea can be used to interpret the present result. Stroking activates low-threshold mechano-sensitive spinal neurons, wide dynamic range spinal neurons including pruritogen-sensitive spinal neurons, and dorsal column nuclei to elicit tactile sensation (Abraira and Ginty, 2013; Abraira et al., 2017). Therefore, while stroking enhanced the response to pruritogens during stimulation, the overall sensation elicited by stroking (and presumably by VGLUT3-lineage nerve stimulation) is touch and not itch. In line with this idea, optogenetic VGLUT3-lineage nerve stimulation alone did not elicit scratching.

Additionally, the pharmacological silencing of $\mathrm{TLR}^{+} \mathrm{A}$ fibers diminished the enhancing effect of stroking with the thick brush during the chloroquine-evoked response. On the other hand, the optical silencing of VGLUT3-lineage nerves did not have such an effect. These data indicate that the enhancement of chloroquine-evoked response by stroking with the thick brush may be mediated by TLR5 ${ }^{+}$A-fibers rather than VGLUT3-lineage nerves.

This study has shown that gentle skin stroking can reduce itch signaling in the spinal cord through the activation of VGLUT3-lineage sensory nerves. The findings reported here shed new light on the role of VGLUT3-lineage LTMRs in itch processing.

\section{References}

Abraira VE, Ginty DD (2013) The sensory neurons of touch. Neuron 79:618-639.

Abraira VE, Kuehn ED, Chirila AM, Springel MW, Toliver AA, Zimmerman AL, Orefice LL, Boyle KA, Bai L, Song BJ, Bashista KA, O’Neill TG, Zhuo J, Tsan C, Hoynoski J, Rutlin M, Kus L, Niederkofler V, Watanabe M, Dymecki SM, et al. (2017) The cellular and synaptic architecture of the mechanosensory dorsal horn. Cell 168:295-310.e9.

Acton D, Ren X, Di Costanzo S, Dalet A, Bourane S, Bertocchi I, Eva C, Goulding M (2019) Spinal neuropeptide Y1 receptor-expressing neurons form an essential excitatory pathway for mechanical itch. Cell Rep 28:625-639.e6.

Akiyama T, Merrill AW, Carstens MI, Carstens E (2009) Activation of superficial dorsal horn neurons in the mouse by a PAR-2 agonist and 5-HT: potential role in itch. J Neurosci 29:6691-6699.

Akiyama T, Tominaga M, Carstens MI, Carstens EE (2012) Site-dependent and state-dependent inhibition of pruritogen-responsive spinal neurons by scratching. Eur J Neurosci 36:2311-2316.

Akiyama T, Tominaga M, Takamori K, Iodi Carstens M, Carstens E (2014a) Role of spinal bombesin-responsive neurons in non-histaminergic itch. J Neurophysiol 112:2283-2289.

Akiyama T, Tominaga M, Takamori K, Carstens MI, Carstens E (2014b) Roles of glutamate, substance $\mathrm{P}$, and gastrin-releasing peptide as spinal neurotransmitters of histaminergic and nonhistaminergic itch. Pain 155:80-92.

Bai L, Lehnert BP, Liu J, Neubarth NL, Dickendesher TL, Nwe PH, Cassidy C, Woodbury CJ, Ginty DD (2015) Genetic identification of an expansive mechanoreceptor sensitive to skin stroking. Cell 163:1783-1795.

Bourane S, Duan B, Koch SC, Dalet A, Britz O, Garcia-Campmany L, Kim E, Cheng L, Ghosh A, Ma Q, Goulding M (2015) Gate control of mechanical itch by a subpopulation of spinal cord interneurons. Science 350:550554.

Cevikbas F, Wang X, Akiyama T, Kempkes C, Savinko T, Antal A, Kukova G, Buhl T, Ikoma A, Buddenkotte J, Soumelis V, Feld M, Alenius H, Dillon SR, Carstens E, Homey B, Basbaum A, Steinhoff M (2014) A sensory neuron-expressed IL-31 receptor mediates T helper cell-dependent itch: involvement of TRPV1 and TRPA1. J Allergy Clin Immunol 133:448-460.

Cuellar JM, Antognini JF, Carstens E (2004) An in vivo method for recording single unit activity in lumbar spinal cord in mice anesthetized with a volatile anesthetic. Brain Res Brain Res Protoc 13:126-134.

Davidson S, Zhang X, Khasabov SG, Simone DA, Giesler GJ Jr (2009) Relief of itch by scratching: state-dependent inhibition of primate spinothalamic tract neurons. Nat Neurosci 12:544-546.

Davidson S, Zhang X, Khasabov SG, Moser HR, Honda CN, Simone DA, Giesler GJ Jr (2012) Pruriceptive spinothalamic tract neurons: physiological properties and projection targets in the primate. J Neurophysiol 108:1711-1723.

Draxler P, Honsek SD, Forsthuber L, Hadschieff V, Sandkuhler J (2014) VGluT3(+) primary afferents play distinct roles in mechanical and cold hypersensitivity depending on pain etiology. J Neurosci 34:12015-12028.

Feng J, Luo J, Yang P, Du J, Kim BS, Hu H (2018) Piezo2 channel-Merkel cell signaling modulates the conversion of touch to itch. Science 360:530533.

Fremeau RT Jr, Voglmaier S, Seal RP, Edwards RH (2004) VGLUTs define subsets of excitatory neurons and suggest novel roles for glutamate. Trends Neurosci 27:98-103.

Griffith TN, Docter TA, Lumpkin EA (2019) Tetrodotoxin-sensitive sodium channels mediate action potential firing and excitability in menthol-sensitive Vglut3-lineage sensory neurons. J Neurosci 39:7086-7101.

Grimes WN, Seal RP, Oesch N, Edwards RH, Diamond JS (2011) Genetic targeting and physiological features of VGLUT3 $^{+}$amacrine cells. Vis Neurosci 28:381-392.

Gursul D, Goksan S, Hartley C, Mellado GS, Moultrie F, Hoskin A, Adams E, Hathway G, Walker S, McGlone F, Slater R (2018) Stroking modulates noxious-evoked brain activity in human infants. Curr Biol 28:R1380R1381.

Habig K, Schanzer A, Schirner W, Lautenschlager G, Dassinger B, Olausson H, Birklein F, Gizewski ER, Kramer HH (2017) Low threshold unmyelinated mechanoafferents can modulate pain. BMC Neurol 17:184.

Hayani K, Weiss M, Weisshaar E (2016) Clinical findings and provision of care in haemodialysis patients with chronic itch: new results from the German Epidemiological Haemodialysis Itch Study. Acta Derm Venereol 96:361-366.

Hillman P, Wall PD (1969) Inhibitory and excitatory factors influencing the receptive fields of lamina 5 spinal cord cells. Exp Brain Res 9:284-306.

Jinks SL, Carstens E (2000) Superficial dorsal horn neurons identified by intracutaneous histamine: chemonociceptive responses and modulation by morphine. J Neurophysiol 84:616-627.

Kambrun C, Roca-Lapirot O, Salio C, Landry M, Moqrich A, Le Feuvre Y (2018) TAFA4 reverses mechanical allodynia through activation of GABAergic transmission and microglial process retraction. Cell Rep 22:2886-2897.

Kardon AP, Polgar E, Hachisuka J, Snyder LM, Cameron D, Savage S, Cai X, Karnup S, Fan CR, Hemenway GM, Bernard CS, Schwartz ES, Nagase H, Schwarzer C, Watanabe M, Furuta T, Kaneko T, Koerber HR, Todd AJ, Ross SE (2014) Dynorphin acts as a neuromodulator to inhibit itch in the dorsal horn of the spinal cord. Neuron 82:573-586.

Le Bars D (2002) The whole body receptive field of dorsal horn multireceptive neurones. Brain Res Brain Res Rev 40:29-44.

Leknes S, Tracey I (2008) A common neurobiology for pain and pleasure. Nat Rev Neurosci 9:314-320.

Li L, Rutlin M, Abraira VE, Cassidy C, Kus L, Gong S, Jankowski MP, Luo W, Heintz N, Koerber HR, Woodbury CJ, Ginty DD (2011) The 
functional organization of cutaneous low-threshold mechanosensory neurons. Cell 147:1615-1627.

Liljencrantz J, Strigo I, Ellingsen DM, Kramer HH, Lundblad LC, Nagi SS, Leknes S, Olausson H (2017) Slow brushing reduces heat pain in humans. Eur J Pain 21:1173-1185.

Loken LS, Wessberg J, Morrison I, McGlone F, Olausson H (2009) Coding of pleasant touch by unmyelinated afferents in humans. Nat Neurosci 12:547-548.

Lou S, Duan B, Vong L, Lowell BB, Ma Q (2013) Runx1 controls terminal morphology and mechanosensitivity of VGLUT3-expressing C-mechanoreceptors. J Neurosci 33:870-882.

Lu Y, Perl ER (2003) A specific inhibitory pathway between substantia gelatinosa neurons receiving direct C-fiber input. J Neurosci 23:8752-8758.

Ma Q (2010) Labeled lines meet and talk: population coding of somatic sensations. J Clin Invest 120:3773-3778.

Madisen L, Zwingman TA, Sunkin SM, Oh SW, Zariwala HA, Gu H, Ng LL, Palmiter RD, Hawrylycz MJ, Jones AR, Lein ES, Zeng H (2010) A robust and high-throughput Cre reporting and characterization system for the whole mouse brain. Nat Neurosci 13:133-140.

Madisen L, Mao T, Koch H, Zhuo JM, Berenyi A, Fujisawa S, Hsu YW, Garcia AJ, Gu X, Zanella S, Kidney J, Gu H, Mao Y, Hooks BM, Boyden ES, Buzsáki G, Ramirez JM, Jones AR, Svoboda K, Han X, et al. (2012) A toolbox of Cre-dependent optogenetic transgenic mice for light-induced activation and silencing. Nat Neurosci 15:793-802.

McIlwrath SL, Lawson JJ, Anderson CE, Albers KM, Koerber HR (2007) Overexpression of neurotrophin-3 enhances the mechanical response properties of slowly adapting type 1 afferents and myelinated nociceptors. Eur J Neurosci 26:1801-1812.

Melzack R, Wall PD (1965) Pain mechanisms: a new theory. Science 150:971-979.

Mishra SK, Hoon MA (2013) The cells and circuitry for itch responses in mice. Science 340:968-971.

Mochizuki H, Shevchenko A, Nattkemper LA, Valdes-Rodriguez R, Yosipovitch G (2017) Suppression of scratching-induced pleasurable sensation by compression nerve blocking and its association with itch relief. Itch (Phila) 2:e7.

Nishida K, Takechi K, Akiyama T, Carstens MI, Carstens E (2013) Scratching inhibits serotonin-evoked responses of rat dorsal horn neurons in a site- and state-dependent manner. Neuroscience 250:275-281.

Pan H, Fatima M, Li A, Lee H, Cai W, Horwitz L, Hor CC, Zaher N, Cin M, Slade H, Huang T, Xu XZ, Duan B (2019) Identification of a spinal circuit for mechanical and persistent spontaneous itch. Neuron 103:1135-1149. e36.
Ross SE, Mardinly AR, McCord AE, Zurawski J, Cohen S, Jung C, Hu L, Mok SI, Shah A, Savner EM, Tolias C, Corfas R, Chen S, Inquimbert P, Xu Y, McInnes RR, Rice FL, Corfas G, Ma Q, Woolf CJ, et al. (2010) Loss of inhibitory interneurons in the dorsal spinal cord and elevated itch in Bhlhb5 mutant mice. Neuron 65:886-898.

Roudaut Y, Lonigro A, Coste B, Hao J, Delmas P, Crest M (2012) Touch sense: functional organization and molecular determinants of mechanosensitive receptors. Channels (Austin) 6:234-245.

Sabroe R, Greaves MW (2004) What is urticaria? Anatomical, physiological, and histological. Urticaria Angioedema 1. In: Greaves MW, Kaplan AP, eds. Urticaria and Angioedema. New York: Marcel Dekker Inc., pp 1-50.

Sakai K, Akiyama T (2019) Disinhibition of touch-evoked itch in a mouse model of psoriasis. J Invest Dermatol 139:1407-1410.

Salter MW, Henry JL (1990) Differential responses of nociceptive vs. nonnociceptive spinal dorsal horn neurones to cutaneously applied vibration in the cat. Pain 40:311-322.

Seal RP, Wang X, Guan Y, Raja SN, Woodbury CJ, Basbaum AI, Edwards RH (2009) Injury-induced mechanical hypersensitivity requires C-low threshold mechanoreceptors. Nature 462:651-655.

Shaikh S, Nagi SS, McGlone F, Mahns DA (2015) Psychophysical investigations into the role of low-threshold C fibres in non-painful affective processing and pain modulation. PLoS One 10:e0138299.

Stander S, Weisshaar E, Mettang T, Szepietowski JC, Carstens E, Ikoma A, Bergasa NV, Gieler U, Misery L, Wallengren J, Darsow U, Streit M, Metze D, Luger TA, Greaves MW, Schmelz M, Yosipovitch G, Bernhard JD (2007) Clinical classification of itch: a position paper of the International Forum for the Study of Itch. Acta Derm Venereol 87:291294.

Sun YG, Zhao ZQ, Meng XL, Yin J, Liu XY, Chen ZF (2009) Cellular basis of itch sensation. Science 325:1531-1534.

Wetzel C, Hu J, Riethmacher D, Benckendorff A, Harder L, Eilers A, Moshourab R, Kozlenkov A, Labuz D, Caspani O, Erdmann B, Machelska H, Heppenstall PA, Lewin GR (2007) A stomatin-domain protein essential for touch sensation in the mouse. Nature 445:206-209.

Woodbury CJ, Koerber HR (2007) Central and peripheral anatomy of slowly adapting type I low-threshold mechanoreceptors innervating trunk skin of neonatal mice. J Comp Neurol 505:547-561.

Xu ZZ, Kim YH, Bang S, Zhang Y, Berta T, Wang F, Oh SB, Ji RR (2015) Inhibition of mechanical allodynia in neuropathic pain by TLR5-mediated A-fiber blockade. Nat Med 21:1326-1331.

Yosipovitch G, Greaves MW, Schmelz M (2003) Itch. Lancet 361:690-694. 